\title{
Synchronized reneging in queueing systems with vacations
}

\author{
Ivo Adan • Antonis Economou • Stella Kapodistria
}

Received: 7 July 2008 / Revised: 23 February 2009 / Published online: 4 April 2009

(C) The Author(s) 2009. This article is published with open access at Springerlink.com

\begin{abstract}
In this paper we present a detailed analysis of queueing models with vacations and impatient customers, where the source of impatience is the absence of the server. Instead of the standard assumption that customers perform independent abandonments, we consider situations where customers abandon the system simultaneously. This is, for example, the case in remote systems where customers may decide to abandon the system, when a transport facility becomes available.
\end{abstract}

Keywords Queueing · Vacations · Reneging - Impatient customers ·

Synchronization $\cdot q$-hypergeometric series $\cdot$ Mean value analysis $\cdot$ Stationary distribution · Fluid limit

Mathematics Subject Classification (2000) 90B22 $60 \mathrm{~K} 25$

\section{Introduction}

Queueing systems with reneging (i.e., impatient customers) have been studied extensively. The main assumption in the literature is that customers perform independent

I. Adan $(\bowtie)$

Department of Mathematics and Computer Science, Technische Universiteit Eindhoven, P.O. Box 513, 5600 MB Eindhoven, The Netherlands

e-mail: iadan@win.tue.nl

A. Economou $\cdot$ S. Kapodistria

Department of Mathematics, University of Athens Panepistemiopolis, 15784 Athens, Greece

A. Economou

e-mail: aeconom@math.uoa.gr

S. Kapodistria

e-mail: stellakap@math.uoa.gr 
abandonments, that is, each one of them sets an impatience clock and abandons the system as soon as the clock expires. For Markovian models, this type of abandonments introduces state-inhomogeneous transition rate matrices, which complicates the computation of the performance measures. For non-Markovian models, the basic idea is to use the methodology from the study of the $M / G / \infty$ queue. In both cases, however, it seems fair to say that most of the models are analytically intractable.

The study of queueing systems with impatient customers goes back at least to the pioneering papers of Palm $[19,20]$ who studied the $M / M / c$ queue, where the customers have independent exponentially distributed impatience times. Subsequently, Daley [7], Takacs [22] and Baccelli et al. [5] considered various queueing models with general service and/or inter-arrival times and more involved abandonment schemes.

More recently, Boxma and de Waal [6] studied the $M / M / c$ queue with generally distributed impatience times, while Altman and Borovkov [2] investigated the stability issue in a retrial queue with impatient customers. In all the aforementioned works, customers become impatient due to the long waiting time already experienced, although the server provides continuously service. The study of reneging within the class of queueing systems with vacations is a new endeavor. Although, there exists a significant number of papers and books on vacation queueing systems (see, e.g., [23] and [24]), the reneging feature has not yet received much attention. Only recently, Altman and Yechiali [3] and Yechiali [25] considered systems with vacations, where the cause of the impatience is the absence of the server. The authors assume that the customers perform independent abandonments, whenever the server is unavailable.

In the present paper, we study two models with vacations, where the customers are impatient but they perform synchronized abandonments. These models are motivated by remote systems where customers have to wait for a certain transport facility to abandon the system. Then, whenever the facility visits the system, the present customers decide whether to leave the system or not. Therefore, we have synchronized departures for some of the customers.

The first model is the single-server queue with multiple vacations, where customers decide whether to abandon the system or not when the vacation periods finish. In the second model, we suppose that the abandonments epochs occur according to a Poisson process during vacation periods. At the abandonment epochs, every present customer remains in the system with probability $q$ or abandons the system with probability $p=1-q$, independently of the others. The analysis of this model extends the analysis of Altman and Yechiali [3], in the framework of synchronized abandonments. The new feature of these models with synchronization is the presence of binomial type jumps at the abandonment epochs. Similar models with binomial type transitions have been recently studied by Economou [9], Artalejo et al. [4], Economou and Fakinos [10] and Economou and Kapodistria [11].

The paper is organized as follows. In Sect. 2, we describe the dynamics of the models. In Sect. 3 we first study the Markovian cases and present, separately, the stationary analysis of the two models. We also obtain more explicit results under various limiting regimes concerning the parameters of the models. In Sects. 4 and 5 we proceed with the non-Markovian cases, assuming general service and vacation time distributions. More specifically, in Sect. 4 we carry out a mean value analysis 
of the two models, while in Sect. 5 we study their stationary distributions by using a generating function approach. In Sect. 6 we present several numerical results that illustrate the effect of the various parameters on the performance measures of the model. The paper concludes with Sect. 7, where we discuss possible generalizations and extensions.

\section{Model description}

We consider a queueing system where customers arrive one by one according to a Poisson process at rate $\lambda$. Service is provided by a single server who can be in one of two modes: on (active) or off (non-active-on vacation). Customers are served singly when the server is on, while no service is provided when the server is off. The service times are generally distributed according to a distribution $B(t)$, having Laplace-Stieltjes transform (LST) $\tilde{B}(s)=E\left(e^{-s B}\right)$ and finite first and second moments $E(B)$ and $E\left(B^{2}\right)$, where the random variable $B$ represents the service time. The residual (or equilibrium) service time is denoted by $B_{\mathrm{e}}$, the distribution $B_{\mathrm{e}}(t)$ of which is given by

$$
B_{\mathrm{e}}(t)=\frac{\int_{0}^{t}(1-B(u)) d u}{E(B)} .
$$

There is infinite waiting room. Whenever the system becomes empty, the server begins a vacation. We assume multiple vacations, i.e., if the system is still empty at the end of a vacation, the server takes another one. If, on the contrary, there is at least one waiting customer at the end of a vacation, the server starts again to provide service. The vacation times are generally distributed according to a distribution $V(t)$, having LST $\tilde{V}(s)=E\left(e^{-s V}\right)$ and finite first and second moments $E(V)$ and $E\left(V^{2}\right)$, where the random variable $V$ represents the vacation time. The residual vacation time is denoted by $V_{\mathrm{e}}$ with distribution

$$
V_{\mathrm{e}}(t)=\frac{\int_{0}^{t}(1-V(u)) d u}{E(V)},
$$

and LST

$$
\tilde{V}_{\mathrm{e}}(s)=\frac{1-\tilde{V}(s)}{E(V) s} .
$$

Regarding the abandonments we consider two models:

- Unique Abandonment Epoch (UAE): Every time the server finishes a vacation, every present customer decides whether to stay in the system with probability $q$ or to abandon it with probability $p=1-q$, independently of the others.

- Multiple Abandonment Epochs (MAE): During server vacations, abandonment epochs occur according to a Poisson process with rate $\zeta$. At these epochs, every present customer remains in the system with probability $q$ or abandons the system with probability $p=1-q$, independently of the others. 
Hence, in either model, the number of customers is reduced according to a binomial distribution at every abandonment epoch. However, the analysis of the UAE model turns out to be much easier than the one of the MAE model. For this reason, in what follows, we describe briefly the results for the UAE model and we provide more details for the analysis of the MAE model.

We are interested in the equilibrium behavior of the model, so we need to establish first the stability condition. For the pure vacation model $(p=0)$ the stability condition is (see, e.g., [23])

$$
\rho=\lambda E(B)<1 .
$$

Hence, the above condition is sufficient for the stability of the UAE and MAE models. It is also necessary, since the system behaves as a standard $M / G / 1$ queue while the server is active. Clearly, the only exception is the degenerate case $p=1$. Then condition (2.2) is not required for stability. Throughout the paper we assume the validity of condition (2.2).

\section{Markovian models: equilibrium distribution}

We consider the two models described in Sect. 2, where both the service and vacation time distributions are exponential with rates $\mu$ and $\gamma$, respectively. The traffic intensity is now $\rho=\frac{\lambda}{\mu}$. Then, each system can be described by a continuous-time Markov chain $\{(L(t), I(t)), t \geq 0\}$, with state space $\{(0,0)\} \cup\{(n, i): i=0,1, n=1,2, \ldots\}$, where $L(t)$ is the number of customers in the system at time $t$ and $I(t)$ expresses the mode of the server at time $t$ (more explicitly, it is equal to 1 if the server is on at that time $t$, and 0 otherwise). Below, we focus on the determination of the equilibrium distribution of the Markov chain $\{(L(t), I(t)), t \geq 0\}$. The two models are treated separately.

To this end, let $\{\pi(n, i): i=0,1$ and $n \geq i\}$, denote the equilibrium distribution. We define the probability generating functions (PGFs) $\Pi_{0}(z)$ and $\Pi_{1}(z)$ of the equilibrium distribution by

$$
\Pi_{0}(z)=\sum_{n=0}^{\infty} \pi(n, 0) z^{n} \quad \text { and } \quad \Pi_{1}(z)=\sum_{n=1}^{\infty} \pi(n, 1) z^{n}, \quad|z| \leq 1 .
$$

We also set $L, I$ and $S$ to be random variables representing the number of customers in the system, the state of the server and the sojourn time of a customer, when the system is in equilibrium. We also denote by $p_{i}=P(I=i)$ the equilibrium probability that the server is in state $i, i=0,1$.

\subsection{Equilibrium distribution of the UAE model}

Figure 1 shows the state-transition diagram for the UAE model. The set of balance equations is given as follows:

$$
\lambda \pi(0,0)=\mu \pi(1,1)+\gamma \sum_{j=1}^{\infty}\left(\begin{array}{l}
j \\
j
\end{array}\right) p^{j} \pi(j, 0),
$$




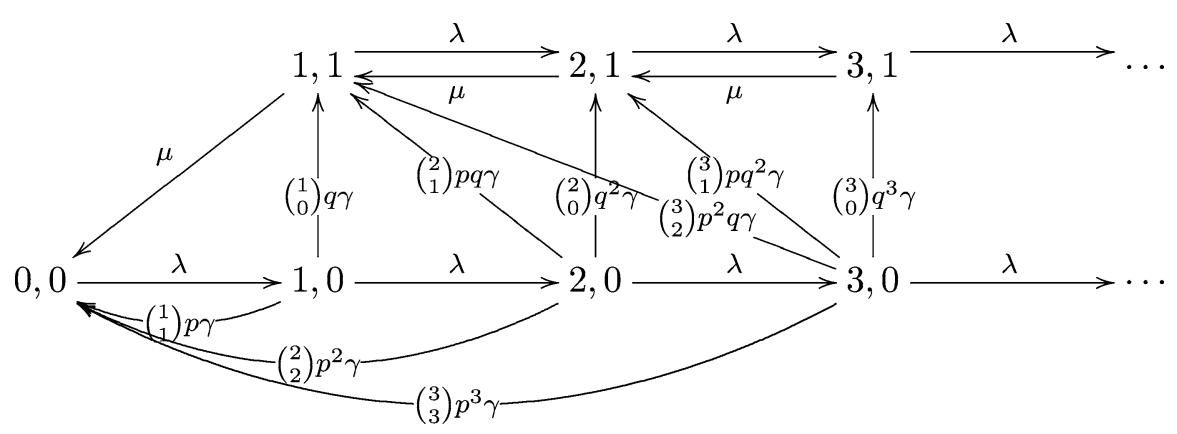

Fig. 1 State-transition diagram for the UAE model

$$
\begin{aligned}
(\lambda+\gamma) \pi(n, 0) & =\lambda \pi(n-1,0), \quad n \geq 1, \\
(\lambda+\mu) \pi(1,1)= & \mu \pi(2,1)+\gamma \sum_{j=1}^{\infty}\left(\begin{array}{c}
j \\
j-1
\end{array}\right) q p^{j-1} \pi(j, 0), \\
(\lambda+\mu) \pi(n, 1)= & \mu \pi(n+1,1)+\lambda \pi(n-1,1) \\
& +\gamma \sum_{j=n}^{\infty}\left(\begin{array}{c}
j \\
j-n
\end{array}\right) q^{n} p^{j-n} \pi(j, 0), \quad n \geq 2 .
\end{aligned}
$$

Provided (2.2), this set of equations, together with the normalization equation

$$
\pi(0,0)+\sum_{n=1}^{\infty}(\pi(n, 0)+\pi(n, 1))=1
$$

has a unique solution. This solution is presented in the next theorem.

Theorem 3.1 Provided (2.2), the equilibrium state distribution $\pi(n, i)$ is given by

$$
\begin{aligned}
& \pi(0,0)=\frac{\gamma}{\gamma+\lambda} \times \frac{1-\rho}{1-\rho p}, \\
& \pi(n, 0)=\pi(0,0)\left(\frac{\lambda}{\lambda+\gamma}\right)^{n}, \quad n \geq 0, \\
& \pi(n, 1)=\left\{\begin{array}{ll}
\pi(0,0) \frac{(\lambda+\gamma) q}{\gamma+(\lambda-\mu) q}\left(\rho^{n}-\left(\frac{\lambda q}{\gamma+\lambda q}\right)^{n}\right), & \text { if } \gamma \neq(\mu-\lambda) q \\
\pi(0,0)(\rho p+q) n \rho^{n}, & \text { if } \gamma=(\mu-\lambda) q
\end{array}, \quad n \geq 1 .\right.
\end{aligned}
$$

Proof By iterating (3.2) we obtain (3.6) yielding

$$
\Pi_{0}(z)=\frac{\lambda+\gamma}{\lambda+\gamma-\lambda z} \pi(0,0) .
$$


By multiplying (3.3) by $z$ and (3.4) by $z^{n}$ and adding for all $n=1,2, \ldots$, we obtain

$$
\begin{aligned}
(\lambda+\mu) \Pi_{1}(z)= & \lambda z \Pi_{1}(z)+\frac{\mu}{z}\left(\Pi_{1}(z)-z \pi(1,1)\right) \\
& +\gamma \Pi_{0}(p+q z)-\gamma \Pi_{0}(p) .
\end{aligned}
$$

Solving (3.9) for $\Pi_{1}(z)$ and plugging (3.8), while using (3.1), yields

$$
\Pi_{1}(z)=\frac{q \rho z(\gamma+\lambda)}{(\gamma+\lambda q(1-z))(1-\rho z)} \pi(0,0) .
$$

Expanding (3.10), for the case $\gamma \neq(\mu-\lambda) q$, in partial fractions and using the geometric series leads to the upper branch of (3.7). For the case $\gamma=(\mu-\lambda) q$, expanding $(1-\rho z)^{-2}$ in power series and equating the coefficients of $z^{n}$ yields the other branch of (3.7). Finally, (3.5) follows from the normalization equation.

Remark 3.1 From (3.8) and (3.10) we obtain the equilibrium mean number of customers in the system given as

$$
\begin{aligned}
E(L) & =\sum_{n=1}^{\infty} n(\pi(n, 0)+\pi(n, 1)) \\
& =\Pi_{0}^{\prime}(1)+\Pi_{1}^{\prime}(1) \\
& =\pi(0,0) \frac{\lambda+\gamma}{\gamma} \times \frac{\lambda}{\gamma}+\pi(0,0) \frac{(\lambda+\gamma) q \rho}{\gamma(1-\rho)} \times \frac{\gamma(1-\rho)+\rho \gamma+\lambda q(1-\rho)}{\gamma(1-\rho)} \\
& =p_{0} \frac{\lambda}{\gamma}+p_{1} \frac{\gamma+\lambda q(1-\rho)}{\gamma(1-\rho)} .
\end{aligned}
$$

This result can be alternatively derived using the mean value approach, as shown in Sect. 4.

Remark 3.2 Let $X(\alpha)$ denote an exponential random variable with rate $\alpha$ and $Y(j, \alpha)$ denote an Erlang random variable consisting of $j$ phases with rate $\alpha$. Let us consider a tagged arriving customer. Then, by the PASTA property, he finds the system in state $(n, i)$ with probability $\pi(n, i)$. If he finds the system in state $(n, 1)$, then his sojourn time is $Y(n+1, \mu)$. If he finds the system in state $(n, 0)$, then with probability $p$ his sojourn time will be $X(\gamma)$, since the tagged customer abandons the system, and with probability $\left(\begin{array}{c}n \\ j\end{array}\right) p^{n-j} q^{j+1}$ his sojourn time will be $X(\gamma)+Y(j+1, \mu)$, for $j=0,1, \ldots, n$, where the random variables $X(\gamma)$ and $Y(j+1, \mu)$ are independent. Hence, by using the geometric form of the equilibrium distribution (3.5)-(3.7), we have that the LST of the sojourn time $\tilde{S}(s)=E\left(e^{-s S}\right)$ can be represented as

$$
\begin{aligned}
\tilde{S}(s)= & p_{0} p \frac{\gamma}{\gamma+s}+p_{0} q \frac{\gamma}{\gamma+s} \times \frac{\gamma \mu}{\gamma \mu+(\gamma+q \lambda) s} \\
& +p_{1} \frac{\mu-\lambda}{\mu-\lambda+s} \times \frac{\gamma \mu}{\gamma \mu+(\gamma+q \lambda) s} .
\end{aligned}
$$


This shows that the sojourn time $S$ is a mixture of $X(\gamma), X(\gamma)+X\left(\frac{\gamma \mu}{\gamma+q \lambda}\right)$, $X(\mu-\lambda)+X\left(\frac{\gamma \mu}{\gamma+q \lambda}\right)$ with mixing probabilities $p_{0} p, p_{0} q$ and $p_{1}$, respectively.

\subsection{Equilibrium distribution of the MAE model}

In this case the state-transition diagram is given in Fig. 2. The set of balance equations for this model is given as follows:

$$
\begin{aligned}
& (\lambda+\zeta) \pi(0,0)=\mu \pi(1,1)+\zeta \sum_{j=0}^{\infty}\left(\begin{array}{l}
j \\
j
\end{array}\right) p^{j} q^{0} \pi(j, 0), \\
& \begin{aligned}
(\lambda+\gamma+\zeta) \pi(n, 0)=\lambda \pi(n-1,0) \\
\quad+\zeta \sum_{j=n}^{\infty}\left(\begin{array}{c}
j \\
j-n
\end{array}\right) p^{j-n} q^{n} \pi(j, 0), \quad n \geq 1, \\
(\lambda+\mu) \pi(1,1)=\gamma \pi(1,0)+\mu \pi(2,1), \\
(\lambda+\mu) \pi(n, 1)=\gamma \pi(n, 0)+\lambda \pi(n-1,1)+\mu \pi(n+1,1), \quad n \geq 2 .
\end{aligned}
\end{aligned}
$$

Note that in the balance equations (3.11) and (3.12) we included the pseudotransitions $(n, 0) \rightarrow(n, 0)$ with rates $\zeta\left(\begin{array}{l}n \\ n\end{array}\right) p^{n-n} q^{n}=\zeta q^{n}$, which correspond to epochs in the Poisson abandonment process where all customers remain in the system, i.e., no abandonments occur. This simplifies the presentation of the balance equations.

In Theorem 3.2 of this section we will determine the equilibrium probability $\pi(0,0)$ and the equilibrium PGF $\Pi_{i}(z), i=0,1$, in the form of infinite series of finite products. These series can be expressed compactly in terms of $q$-hypergeometric series (also known as basic hypergeometric series). Moreover, we will see that the theory of $q$-hypergeometric series easily yields interesting results for some limiting regimes.

There exists a rich theory on the class of $q$-hypergeometric series and their $q$-calculus, which enables fast calculations and simplifications. In the queueing theory literature several applications can be found of the hypergeometric series (see, e.g., [14] and [18]), but only a few of $q$-hypergeometric series (see e.g. [15-17]). Therefore, for the sake of completeness, we summarize below the basic definitions

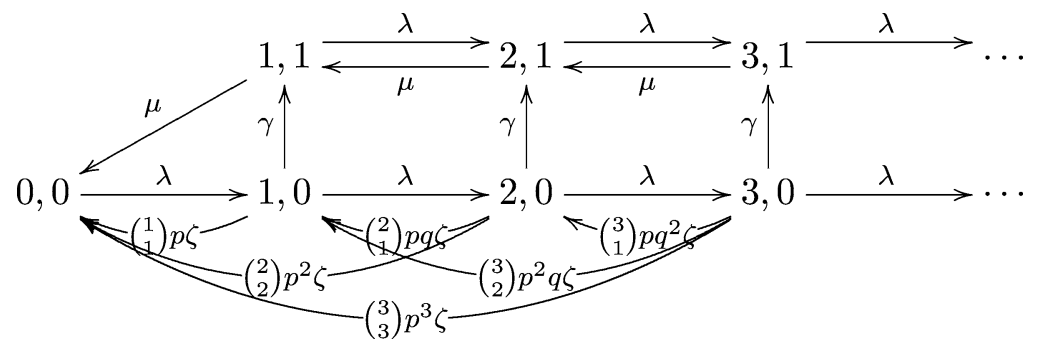

Fig. 2 State-transition diagram for the MAE model 
about $q$-hypergeometric series (for details, see the reference book of Gasper and Rahman [13], Chaps. 1-3 and Appendices I-III).

The $q$-hypergeometric series are series of the form $\sum_{n=0}^{\infty} c_{n}$ where $c_{0}=1$ and $\frac{c_{n+1}}{c_{n}}$ is a rational function of $q^{n}$ for a deformation parameter $|q|<1$. They were initially introduced by Heine who developed their basic theory, following Gauss' fundamental paper on hypergeometric series. Observing that the ratio $\frac{c_{n+1}}{c_{n}}$, being rational in $q^{n}$, can be written in the form

$$
\frac{c_{n+1}}{c_{n}}=\frac{\left(1-a_{1} q^{n}\right)\left(1-a_{2} q^{n}\right) \cdots\left(1-a_{r} q^{n}\right)}{\left(1-q^{n+1}\right)\left(1-b_{1} q^{n}\right) \cdots\left(1-b_{s} q^{n}\right)}\left(-q^{n}\right)^{1+s-r} z,
$$

we have that every such series assumes the form

$$
\begin{aligned}
{ }_{r} \phi_{s}\left(\begin{array}{c}
a_{1}, a_{2}, \ldots, a_{r} \\
b_{1}, \ldots, b_{s}
\end{array} ;, z\right) & \equiv{ }_{r} \phi_{s}\left(a_{1}, a_{2}, \ldots, a_{r} ; b_{1}, \ldots, b_{s} ; q, z\right) \\
& :=\sum_{n=0}^{\infty} \frac{\left(a_{1} ; q\right)_{n}\left(a_{2} ; q\right)_{n} \cdots\left(a_{r} ; q\right)_{n}}{(q ; q)_{n}\left(b_{1} ; q\right)_{n} \cdots\left(b_{s} ; q\right)_{n}}\left[(-1)^{n} q^{\left(\begin{array}{c}
n \\
2
\end{array}\right)}\right]^{1+s-r} z^{n},
\end{aligned}
$$

where $(a ; q)_{0}=1$ and $(a ; q)_{n}=(1-a)(1-a q)\left(1-a q^{2}\right) \cdots\left(1-a q^{n-1}\right), n \geq 1$. In the definition of a $q$-series through (3.15) it is assumed that $b_{i} \neq q^{-m}$ for $m=0,1, \ldots$ and $i=1,2, \ldots, s$. This is the standard ${ }_{r} \phi_{s}$ notation for $q$-series. If $0<|q|<1$, the ${ }_{r} \phi_{s}$ series converges absolutely for all $z$ when $r \leq s$ and for $|z|<1$ when $r=s+1$. We use the abbreviation $\left(a_{1}, a_{2}, \ldots, a_{r} ; q\right)_{n}$ to denote the product $\left(a_{1} ; q\right)_{n}\left(a_{2} ; q\right)_{n} \cdots\left(a_{r} ; q\right)_{n}$. The quantity $(a ; q)_{n}$ is referred to as the $q$ shifted factorial. We also define $(a ; q)_{\infty}=\prod_{k=0}^{\infty}\left(1-a q^{k}\right)$ and use the abbreviation $\left(a_{1}, a_{2}, \ldots, a_{r} ; q\right)_{\infty}$ to denote the product $\left(a_{1} ; q\right)_{\infty}\left(a_{2} ; q\right)_{\infty} \cdots\left(a_{r} ; q\right)_{\infty}$. A $q$ calculus has been developed that parallels the theory of hypergeometric functions.

We are now in position to state the main result of this section.

Theorem 3.2 Provided (2.2), the equilibrium state probability of an empty system $\pi(0,0)$ is given by

$$
\begin{aligned}
\pi(0,0) & =\frac{A}{\zeta} \sum_{j=0}^{\infty} \prod_{k=0}^{j} \frac{\zeta}{\gamma+\zeta+\lambda q^{k}} \\
& =\frac{A}{\gamma+\zeta} \frac{(q ; q)_{\infty}}{\left(-\frac{\lambda}{\gamma+\zeta}, \frac{\zeta}{\gamma+\zeta} ; q\right)_{\infty}} 2 \phi_{1}\left(-\frac{\lambda}{\gamma+\zeta}, \frac{\zeta}{\gamma+\zeta} ; 0 ; q, q\right) .
\end{aligned}
$$

The partial PGFs $\Pi_{0}(z)$ and $\Pi_{1}(z)$ are given by

$$
\begin{aligned}
\Pi_{0}(z) & =\frac{A}{\zeta} \sum_{j=0}^{\infty} \prod_{k=0}^{j} \frac{\zeta}{\gamma+\zeta+\lambda q^{k}(1-z)} \\
& =\frac{A}{\gamma+\zeta} \frac{(q ; q)_{\infty}}{\left(-\frac{\lambda(1-z)}{\gamma+\zeta}, \frac{\zeta}{\gamma+\zeta} ; q\right)_{\infty}} 2 \phi_{1}\left(-\frac{\lambda(1-z)}{\gamma+\zeta}, \frac{\zeta}{\gamma+\zeta} ; 0 ; q, q\right)
\end{aligned}
$$




$$
\Pi_{1}(z)=-\frac{A z}{\lambda z+\mu z-\lambda z^{2}-\mu}+\frac{\gamma z}{\lambda z+\mu z-\lambda z^{2}-\mu} \Pi_{0}(z)
$$

where

$$
A=\frac{\gamma(\mu-\lambda)(\gamma+\zeta(1-q))}{\mu \gamma+(\mu-\lambda) \zeta(1-q)} .
$$

The convergence of the series is absolute in $\{z \in \mathbb{C}:|z| \leq 1\}$ and uniform in every compact subset of $\{z \in \mathbb{C}:|z|<1\}$.

Proof Multiplying both sides of (3.11) and (3.12) by $z^{0}$ and $z^{n}$, respectively, and summing them for all $n=0,1,2, \ldots$, we obtain

$$
\begin{aligned}
(\lambda+\gamma+\zeta) \Pi_{0}(z)-\gamma \pi(0,0)= & \mu \pi(1,1)+\lambda z \Pi_{0}(z) \\
& +\zeta \sum_{n=0}^{\infty} \sum_{j=n}^{\infty}\left(\begin{array}{l}
j \\
n
\end{array}\right) p^{j-n} q^{n} \pi(j, 0) z^{n}
\end{aligned}
$$

or

$$
(\lambda+\gamma+\zeta-\lambda z) \Pi_{0}(z)=\gamma \pi(0,0)+\mu \pi(1,1)+\zeta \Pi_{0}(1-q+q z),
$$

which leads to

$$
\Pi_{0}(z)=\frac{\gamma \pi(0,0)+\mu \pi(1,1)}{\gamma+\zeta+\lambda(1-z)}+\frac{\zeta}{\gamma+\zeta+\lambda(1-z)} \Pi_{0}(1-q+q z) .
$$

Furthermore, by multiplying both sides of (3.13) and (3.14) by $z$ and $z^{n}$, respectively, and summing them for all $n=1,2,3, \ldots$ we obtain after some rearrangements that

$$
\Pi_{1}(z)=-\frac{(\gamma \pi(0,0)+\mu \pi(1,1)) z}{\lambda z+\mu z-\lambda z^{2}-\mu}+\frac{\gamma z}{\lambda z+\mu z-\lambda z^{2}-\mu} \Pi_{0}(z) .
$$

By iterating (3.23) and setting

$$
A=\gamma \pi(0,0)+\mu \pi(1,1),
$$

we obtain

$$
\begin{aligned}
\Pi_{0}(z)= & \frac{A}{\zeta} \sum_{j=0}^{n} \prod_{k=0}^{j} \frac{\zeta}{\gamma+\zeta+\lambda q^{k}(1-z)} \\
& +\Pi_{0}\left(1-q^{n+1}+q^{n+1} z\right) \prod_{k=0}^{n} \frac{\zeta}{\gamma+\zeta+\lambda q^{k}(1-z)}, \quad n \geq 0 .
\end{aligned}
$$

By letting $n \rightarrow \infty$ we get

$$
\Pi_{0}(z)=\frac{A}{\zeta} \sum_{j=0}^{\infty} \prod_{k=0}^{j} \frac{\zeta}{\gamma+\zeta+\lambda q^{k}(1-z)},
$$


which is expressed as a $q$-hypergeometric series in the form (3.18). This shows also that the infinite series does converge. We set $z=0$ in (3.27), yielding

$$
\pi(0,0)=\frac{A}{\zeta} \sum_{j=0}^{\infty} \prod_{k=0}^{j} \frac{\zeta}{\gamma+\zeta+\lambda q^{k}},
$$

which can be put in the form (3.16). We set $z=1$ in (3.27), which leads to

$$
\Pi_{0}(1)=\frac{A}{\zeta} \sum_{j=0}^{\infty} \prod_{k=0}^{j} \frac{\zeta}{\gamma+\zeta}=\frac{A}{\gamma} .
$$

Note also that by (3.24), (3.25) and (3.29) we obtain

$$
\Pi_{1}(1)=\frac{\gamma}{\mu-\lambda} \Pi_{0}^{\prime}(1)
$$

To obtain $\Pi_{0}^{\prime}(1)$ multiply (3.23) by $\gamma+\zeta+\lambda(1-z)$, differentiate and take $z \rightarrow 1$. We then have

$$
\Pi_{0}^{\prime}(1)=\frac{\lambda A}{\gamma(\gamma+\zeta(1-q))} .
$$

Equations (3.30) and (3.31) yield

$$
\Pi_{1}(1)=\frac{\lambda A}{(\mu-\lambda)(\gamma+\zeta(1-q))} .
$$

We have now expressed the various quantities of interest and the PGFs $\Pi_{0}(z)$ and $\Pi_{1}(z)$ in terms of the parameters of the model and the parameter $A$. Using (3.29) and (3.32) and the normalization equation we obtain (3.20) which concludes the proof.

Remark 3.3 By differentiating twice (3.23) and once (3.24) and taking $z \rightarrow 1$ we obtain, after some long calculations, the mean number of customers in system. However, as will be shown in Sect. 4, the mean value approach gives the result much more easily.

In the next Sects. 3.3 and 3.4 we consider the fluid limits for the UAE and MAE models. Similar studies have been reported in [1] and [8].

\subsection{Fluid limit of the UAE model}

In this section we study the scaled queue length process in the UAE model as both the arrival rate $\lambda$ and service rate $\mu$ tend to infinity, while keeping the traffic intensity $\rho=\frac{\lambda}{\mu}$ fixed. That is, we consider the sequence of queue length processes $L^{m}(t)$ with arrival rates $\lambda_{m}=m \lambda$, service rates $\mu_{m}=m \mu$ and constant vacation rate $\gamma_{m}=\gamma$, and then we are interested in obtaining the equilibrium distribution of the fluid scaled limit

$$
\bar{L}(t)=\lim _{m \rightarrow \infty} \frac{L^{m}(t)}{m} .
$$


Fig. 3 The fluid scaled sample path of $L^{m}(t)$ in the UAE model as $m \rightarrow \infty$

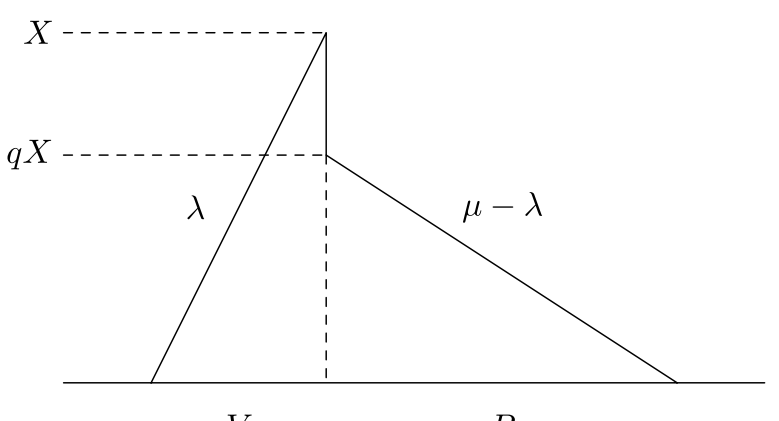

$V$

$P$

The fluid scaled sample path of $L^{m}(t)$ is shown in Fig. 3. Clearly, a cycle first starts with an exponential vacation $V$, at the start of which there is no fluid and during which the amount of fluid increases with rate $\lambda$. At the end of the vacation an exponential amount $X=\lambda V$ accumulated in the queue; a fraction $p$ of $X$ will then immediately abandon and the remainder $q X$ will be drained at rate $\mu-\lambda$, which takes an exponential time $P=q X /(\mu-\lambda)$. When the queue is empty again, the cycle repeats.

Let the random variable $\bar{L}$ denote the amount of fluid in the queue, when the system is in equilibrium. From the memoryless property of exponentials, we immediately obtain

$$
\bar{L} \stackrel{\mathrm{d}}{=} \begin{cases}X & \text { w.p. } \frac{E(V)}{E(V)+E(P)}, \\ q X & \text { w.p. } \frac{E(P)}{E(V)+E(P)},\end{cases}
$$

where

$$
E(V)=\frac{1}{\gamma}, \quad E(P)=\frac{q}{\mu-\lambda} \lambda E(V)=\frac{q \rho}{1-\rho} \frac{1}{\gamma} .
$$

In addition to the above intuitive derivation of (3.33), we prove the following theorem by directly employing (3.5), (3.8) and (3.10).

Theorem 3.3 The LST of $\bar{L}$ is given by

$$
E\left(e^{-s \bar{L}}\right)=\frac{1-\rho}{1-\rho+q \rho} \frac{\frac{\gamma}{\lambda}}{\frac{\gamma}{\lambda}+s}+\frac{q \rho}{1-\rho+q \rho} \frac{\frac{\gamma}{q \lambda}}{\frac{\gamma}{q \lambda}+s} .
$$

Proof We can determine the LST of $\bar{L}$ as the limit of $E\left(e^{-s L^{m} / m}\right)$ as $m \rightarrow \infty$,

$$
\begin{aligned}
E\left(e^{-s \bar{L}}\right) & =\lim _{m \rightarrow \infty} E\left(e^{-s L^{m} / m}\right) \\
& =\lim _{m \rightarrow \infty}\left[\Pi_{0}^{(m)}\left(e^{-s / m}\right)+\Pi_{1}^{(m)}\left(e^{-s / m}\right)\right] \\
& =\lim _{m \rightarrow \infty}\left[\frac{m \lambda+\gamma}{m \lambda+\gamma-m \lambda e^{-s / m}} \frac{\gamma}{\gamma+m \lambda} \frac{1-\rho}{1-p \rho}\right.
\end{aligned}
$$




$$
\begin{aligned}
& \left.+\frac{q \rho e^{-s / m}(\gamma+m \lambda)}{\left(\gamma+m \lambda q\left(1-e^{-s / m}\right)\right)\left(1-\rho e^{-s / m}\right)} \frac{\gamma}{\gamma+m \lambda} \frac{1-\rho}{1-p \rho}\right] \\
= & \lim _{m \rightarrow \infty}\left[\frac{\gamma}{\gamma+m \lambda\left(1-e^{-s / m}\right)} \frac{1-\rho}{1-p \rho}\right. \\
& \left.+\frac{q \rho e^{-s / m} \gamma}{\left(\gamma+m \lambda q\left(1-e^{-s / m}\right)\right)\left(1-\rho e^{-s / m}\right)} \frac{1-\rho}{1-p \rho}\right] .
\end{aligned}
$$

Since

$$
\lim _{m \rightarrow \infty} m\left(1-e^{-s / m}\right)=s,
$$

we obtain

$$
E\left(e^{-s \bar{L}}\right)=\frac{\gamma}{\gamma+\lambda s} \frac{1-\rho}{1-p \rho}+\frac{\gamma}{\gamma+\lambda q s} \frac{q \rho}{1-p \rho} .
$$

\subsection{Fluid limit of the MAE model}

Now we study the scaled queue length process in the MAE model as both the arrival rate $\lambda$ and service rate $\mu$ tend to infinity, while keeping the traffic intensity $\rho=\frac{\lambda}{\mu}$ fixed. So we consider the sequence of queue length processes $L^{m}(t)$ with arrival rates $\lambda_{m}=m \lambda$, service rates $\mu_{m}=m \mu$ and constant vacation rates $\gamma_{m}=\gamma$ and abandonment rates $\zeta_{m}=\zeta$. We are interested in obtaining the equilibrium distribution of the fluid scaled limit

$$
\bar{L}(t)=\lim _{m \rightarrow \infty} \frac{L^{m}(t)}{m} .
$$

The fluid scaled sample path of $L^{m}(t)$ is shown in Fig. 4. The cycle starts with an empty queue and a random number of exponential vacations $V_{1}, \ldots, V_{N}$, each with mean $\frac{1}{\gamma+\zeta}$ and during which the amount of fluid increases with rate $\lambda$. The amount of fluid that is added during $V_{n}$ is $X_{n}=\lambda V_{n}$. The number of vacations is geometrically distributed with success probability $\frac{\gamma}{\zeta+\gamma}$,

$$
P(N=n)=\left(\frac{\gamma}{\zeta+\gamma}\right)^{n-1} \frac{\gamma}{\zeta+\gamma}, \quad n=1,2, \ldots
$$

At the end of each vacation $V_{n}$, a fraction $p$ of the total amount of fluid in the queue will instantly abandon. The only exception is the last vacation $V_{N}$. At the end of

Fig. 4 The fluid scaled sample path of $L^{m}(t)$ in the MAE model as $m \rightarrow \infty$

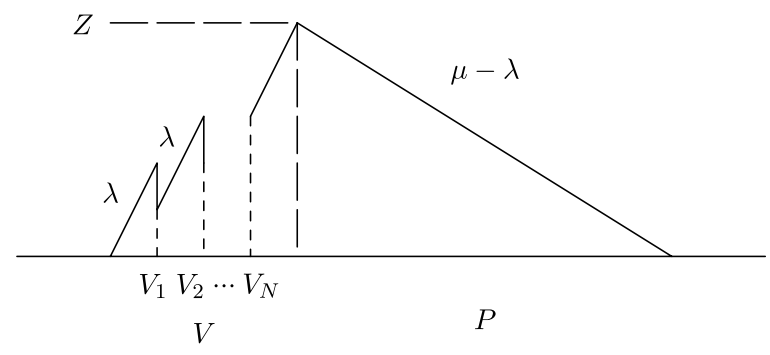


$V_{N}$ no fluid is removed, since this is not an abandonment epoch, but the end of the vacation period. The total amount of fluid in the queue at the end of $V_{N}$ is denoted by the random variable $Z$; so

$$
Z=\sum_{n=1}^{N} q^{N-n} X_{n}
$$

Subsequently the service period starts, during which the queue is drained at rate $\mu-\lambda$. The time required to empty the queue is $P=Z /(\mu-\lambda)$. Finally, when the queue is empty, the cycle repeats.

As before, let the random variable $\bar{L}$ denote the amount of fluid in the queue, when the system is in equilibrium. Further, let $\bar{L}_{i}$ be the amount of fluid in the queue, given that the server is in state $i, i=0,1$. Using the memoryless properties of $N$ and $X_{i}$, we immediately have

$$
\bar{L}_{0} \stackrel{\mathrm{d}}{=} Z=\sum_{n=1}^{N} q^{N-n} X_{n}
$$

Note that $\bar{L}_{1}$ is the amount of fluid that is drained during the residual service period $P_{\mathrm{e}}$, so

$$
\bar{L}_{1}=P_{\mathrm{e}}(\mu-\lambda)=Z_{e}
$$

Hence, by (3.35) and (3.36) we get

$$
\begin{aligned}
E\left(e^{-s \bar{L}_{0}}\right) & =\frac{\gamma}{\zeta} \sum_{k=1}^{\infty} \prod_{n=0}^{k-1} \frac{\zeta}{\gamma+\zeta+\lambda s q^{n}} \\
& =\frac{\gamma}{\gamma+\zeta+s \lambda} 2 \phi_{1}\left(0, q ;-\frac{s \lambda q}{\gamma+\zeta} ; q, \frac{\zeta}{\gamma+\zeta}\right),
\end{aligned}
$$

and

$$
\begin{aligned}
E\left(e^{-s \bar{L}_{1}}\right) & =\frac{1-E\left(e^{-s \bar{L}_{0}}\right)}{E\left(\bar{L}_{0}\right) s} \\
& =\frac{\gamma+\zeta(1-q)}{\lambda} \cdot \frac{1-E\left(e^{-s \bar{L}_{0}}\right)}{s},
\end{aligned}
$$

where we used that

$$
E\left(\bar{L}_{0}\right)=E(Z)=\frac{\lambda}{\gamma+\zeta(1-q)} .
$$

Finally, we have

$$
E\left(e^{-s \bar{L}}\right)=\frac{E(V)}{E(V)+E(P)} E\left(e^{-s \bar{L}_{0}}\right)+\frac{E(P)}{E(V)+E(P)} E\left(e^{-s \bar{L}_{1}}\right),
$$

where $V$ is the total vacation period, i.e., $V=V_{1}+\cdots+V_{N}$ (and so $E(V)=\frac{1}{\gamma}$ ). In addition to this intuitive derivation of the LST of $\bar{L}$, we prove the following theorem by directly employing the expressions (3.17) and (3.19). 
Theorem 3.4 The LST of $\bar{L}$ is given by

$$
\begin{aligned}
E\left(e^{-s \bar{L}}\right)= & \frac{A}{\gamma}{ }_{2} \phi_{1}\left(0, q ; \frac{\zeta q}{\gamma+\zeta} ; q,-\frac{s \lambda}{\gamma+\zeta}\right) \\
& +\frac{A}{\gamma+\zeta(1-q)} \frac{\rho}{1-\rho} 2 \phi_{1}\left(0, q ; \frac{\zeta q^{2}}{\gamma+\zeta} ; q,-\frac{s \lambda}{\gamma+\zeta}\right) \\
= & \frac{A}{\gamma}+\frac{\lambda(\gamma-(\mu-\lambda) s)}{\mu \gamma+(\mu-\lambda) \zeta(1-q)} 2 \phi_{1}\left(0, q ; \frac{\zeta q^{2}}{\gamma+\zeta} ; q,-\frac{s \lambda}{\gamma+\zeta}\right),
\end{aligned}
$$

where the constant $A$ is given by (3.20). Moreover,

$$
E(\bar{L})=\frac{\lambda(\mu-\lambda)}{\mu \gamma+(\mu-\lambda) \zeta p}+\frac{\lambda^{2} \gamma}{(\mu \gamma+(\mu-\lambda) \zeta p)\left(\gamma+\zeta\left(1-q^{2}\right)\right)} .
$$

Proof We determine the LST of $\bar{L}$ as

$$
\begin{aligned}
E\left(e^{-s \bar{L}}\right) & =\lim _{m \rightarrow \infty} E\left(e^{-s \frac{L^{m}}{m}}\right) \\
& =\lim _{m \rightarrow \infty}\left[\Pi_{0}^{(m)}\left(e^{-s / m}\right)+\Pi_{1}^{(m)}\left(e^{-s / m}\right)\right] .
\end{aligned}
$$

From (3.17) we get

$$
\Pi_{0}^{(m)}\left(e^{-s / m}\right)=\frac{A}{\zeta} \sum_{j=0}^{\infty} \prod_{k=0}^{j} \frac{\zeta}{\gamma+\zeta+m \lambda q^{k}\left(1-e^{-s / m}\right)} .
$$

Taking the limit as $m \rightarrow \infty$ and using (3.34) yields

$$
\begin{aligned}
\lim _{m \rightarrow \infty} \Pi_{0}^{(m)}\left(e^{-s / m}\right) & =\frac{A}{\zeta} \sum_{j=0}^{\infty} \prod_{k=0}^{j} \frac{\zeta}{\gamma+\zeta+s \lambda q^{k}} \\
& =\frac{A}{\gamma+\zeta+s \lambda} 2 \phi_{1}\left(0, q ;-\frac{s \lambda q}{\gamma+\zeta} ; q, \frac{\zeta}{\gamma+\zeta}\right) \\
& =\frac{A}{\gamma} 2 \phi_{1}\left(0, q ; \frac{\zeta q}{\gamma+\zeta} ; q,-\frac{s \lambda}{\gamma+\zeta}\right),
\end{aligned}
$$

where the last equality follows from formula (III.2) on p. 359 in [13]. Similarly, from (3.19),

$$
\begin{aligned}
\lim _{m \rightarrow \infty} \Pi_{1}^{(m)}\left(e^{-s / m}\right)= & \lim _{m \rightarrow \infty}\left[-\frac{A e^{-s / m}}{m\left(\lambda e^{-s / m}+\mu e^{-s / m}-\lambda e^{-2 s / m}-\mu\right)}\right. \\
& \left.+\frac{\gamma e^{-s / m}}{m\left(\lambda e^{-s / m}+\mu e^{-s / m}-\lambda e^{-2 s / m}-\mu\right)} \Pi_{0}^{(m)}\left(e^{-s / m}\right)\right] \\
= & \frac{A}{s(\mu-\lambda)}-\frac{\gamma}{s(\mu-\lambda)} \lim _{m \rightarrow \infty} \Pi_{0}^{(m)}\left(e^{-s / m}\right)
\end{aligned}
$$




$$
\begin{aligned}
& =\frac{A}{s(\mu-\lambda)}-\frac{A}{s(\mu-\lambda)} 2_{1} \phi_{1}\left(0, q ; \frac{\zeta q}{\gamma+\zeta} ; q,-\frac{s \lambda}{\gamma+\zeta}\right) \\
& =\frac{A}{\mu-\lambda} \sum_{n=1}^{\infty} \frac{\left(\frac{\lambda}{\gamma+\zeta}\right)^{n}(-s)^{n-1}}{\left(\frac{\zeta q}{\gamma+\zeta} ; q\right)_{n}} \\
& =\frac{A}{\mu-\lambda} \sum_{n=1}^{\infty} \frac{\frac{\lambda}{\gamma+\zeta}\left(\frac{\lambda}{\gamma+\zeta}\right)^{n-1}(-s)^{n-1}}{\left(1-\frac{\zeta q}{\gamma+\zeta}\right)\left(\frac{\zeta q^{2}}{\gamma+\zeta} ; q\right)_{n-1}} \\
& =\frac{A}{\mu-\lambda} \frac{\lambda}{\gamma+\zeta(1-q)} 2 \phi_{1}\left(0, q ; \frac{\zeta q^{2}}{\gamma+\zeta} ; q,-\frac{s \lambda}{\gamma+\zeta}\right) .
\end{aligned}
$$

Finally

$$
\begin{aligned}
E\left(e^{-s \bar{L}}\right)= & \frac{A}{\gamma}{ }_{2} \phi_{1}\left(0, q ; \frac{\zeta q}{\gamma+\zeta} ; q,-\frac{s \lambda}{\gamma+\zeta}\right)+\frac{A}{\gamma+\zeta(1-q)} \frac{\rho}{1-\rho} \\
& \times{ }_{2} \phi_{1}\left(0, q ; \frac{\zeta q^{2}}{\gamma+\zeta} ; q,-\frac{s \lambda}{\gamma+\zeta}\right) \\
= & \frac{A}{\gamma}-\frac{A}{\gamma} \frac{s \lambda}{\gamma+\zeta(1-q)} 2 \phi_{1}\left(0, q ; \frac{\zeta q^{2}}{\gamma+\zeta} ; q,-\frac{s \lambda}{\gamma+\zeta}\right) \\
& +\frac{A}{\gamma+\zeta(1-q)} \frac{\rho}{1-\rho} 2 \phi_{1}\left(0, q ; \frac{\zeta q^{2}}{\gamma+\zeta} ; q,-\frac{s \lambda}{\gamma+\zeta}\right) \\
= & \frac{A}{\gamma}+\left(\frac{A}{\gamma+\zeta(1-q)} \frac{\rho}{1-\rho}-\frac{A}{\gamma} \frac{s \lambda}{\gamma+\zeta(1-q)}\right) \\
& \times{ }_{2} \phi_{1}\left(0, q ; \frac{\zeta q^{2}}{\gamma+\zeta} ; q,-\frac{s \lambda}{\gamma+\zeta}\right) \\
= & \frac{A}{\gamma}+\frac{\lambda(\gamma-(\mu-\lambda) s)}{\mu \gamma+(\mu-\lambda) \zeta(1-q)}{ }_{2} \phi_{1}\left(0, q ; \frac{\zeta q^{2}}{\gamma+\zeta} ; q,-\frac{s \lambda}{\gamma+\zeta}\right) .
\end{aligned}
$$

To obtain the $E(\bar{L})$, we will need two basic results,

$$
\begin{aligned}
& \left.{ }_{2} \phi_{1}\left(0, q ; \frac{\zeta q^{2}}{\gamma+\zeta} ; q,-\frac{s \lambda}{\gamma+\zeta}\right)\right|_{s=0}=1, \\
& \left.\frac{d}{d s}{ }_{2} \phi_{1}\left(0, q ; \frac{\zeta q^{2}}{\gamma+\zeta} ; q,-\frac{s \lambda}{\gamma+\zeta}\right)\right|_{s=0}=-\frac{\lambda}{\gamma+\zeta-\zeta q^{2}}
\end{aligned}
$$

Then, taking the derivative of the LST of $\bar{L}$ and substituting $s=0$ and multiplying by -1 , yields 


$$
\begin{aligned}
E(\bar{L}) & =-\left.\frac{d}{d s}\left(\frac{\lambda(\gamma-(\mu-\lambda) s)}{\mu \gamma+(\mu-\lambda) \zeta(1-q)} 2 \phi_{1}\left(0, q ; \frac{\zeta q^{2}}{\gamma+\zeta} ; q,-\frac{s \lambda}{\gamma+\zeta}\right)\right)\right|_{s=0} \\
& =\frac{\lambda(\mu-\lambda)}{\mu \gamma+(\mu-\lambda) \zeta(1-q)} \times 1+\frac{\lambda \gamma}{\mu \gamma+(\mu-\lambda) \zeta(1-q)} \times \frac{\lambda}{\gamma+\zeta-\zeta q^{2}}
\end{aligned}
$$

which completes the proof of the theorem.

\subsection{Limiting regimes of synchronization in the MAE model}

To emphasize the dependence on the parameters of the MAE model in the rest of this section, we will denote $\pi(n, i), \Pi_{0}(z)$ and $\Pi_{1}(z)$ by $\pi(n, i ; \lambda, \mu, \zeta, p, \gamma)$, $\Pi_{0}(z ; \lambda, \mu, \zeta, p, \gamma)$ and $\Pi_{1}(z ; \lambda, \mu, \zeta, p, \gamma)$ respectively. Note that $\zeta p$ can be thought of as the effective abandonment rate per customer. Indeed the overall abandonment time of a customer is a geometric sum of exponentially distributed random variables with rate $\zeta$; hence it is also exponentially distributed with parameter $\zeta p$. Under this perspective, if we have two models with the same parameters $\lambda, \mu$ and $\gamma$ that differ only in $\zeta$ and $p$, but with $\zeta p=\zeta^{*}$ fixed, we can think that the models have identical arrival rates, service rates, effective abandonment rates per customer and vacation rates and differ only in the 'level of synchronization' $p$. Indeed, the case $p \rightarrow 0^{+}$corresponds to no-synchronization since the customers abandon almost singly the system. On the contrary, the case $p \rightarrow 1^{-}$corresponds to full synchronization since almost all present customers abandon simultaneously.

We are interested in studying the equilibrium behavior of the system for the case where $\lambda, \mu, \zeta^{*}$ and $\gamma$ are kept fixed in the two limiting cases $p \rightarrow 0^{+}\left(q \rightarrow 1^{-}\right)$and $p \rightarrow 1^{-}\left(q \rightarrow 0^{+}\right)$. For the limiting case of no-synchronization we introduce

$$
\begin{aligned}
& \pi^{(1)}(0,0)=\lim _{q \rightarrow 1^{-}} \pi\left(0,0 ; \lambda, \mu, \frac{\zeta^{*}}{1-q}, 1-q, \gamma\right), \\
& \Pi_{i}^{(1)}(z)=\lim _{q \rightarrow 1^{-}} \Pi_{i}\left(z ; \lambda, \mu, \frac{\zeta^{*}}{1-q}, 1-q, \gamma\right), \quad i=0,1,
\end{aligned}
$$

and for the limiting case of full synchronization,

$$
\begin{aligned}
& \pi^{(2)}(0,0)=\lim _{q \rightarrow 0^{+}} \pi\left(0,0 ; \lambda, \mu, \frac{\zeta^{*}}{1-q}, 1-q, \gamma\right), \\
& \Pi_{i}^{(2)}(z)=\lim _{q \rightarrow 0^{+}} \Pi_{i}\left(z ; \lambda, \mu, \frac{\zeta^{*}}{1-q}, 1-q, \gamma\right), \quad i=0,1 .
\end{aligned}
$$

The corresponding results for (3.37)-(3.40) are presented in Theorems 3.5 and 3.6.

To obtain immediately the results, we will use some results of the $q$-theory, concerning the definite $q$-integral of a function on an interval $[0, a]$ which is defined by

$$
\int_{0}^{a} f(t) d_{q} t=a(1-q) \sum_{n=0}^{\infty} f\left(a q^{n}\right) q^{n}
$$


As $q \rightarrow 1^{-}$the $q$-analogues reduce to their standard counterparts. In particular, we have the relationships:

$$
\begin{aligned}
& \lim _{q \rightarrow 1^{-}}(a(1-q) ; q)_{\infty}=e^{-a}, \\
& \lim _{q \rightarrow 1^{-}} \frac{\left(q^{a} s ; q\right)_{\infty}}{(s ; q)_{\infty}}=(1-s)^{-a}, \\
& \lim _{q \rightarrow 1^{-}} \int_{0}^{a} f(t) d_{q} t=\int_{0}^{a} f(t) d t
\end{aligned}
$$

(see [13], (1.3.17), (1.3.19) and (1.11.6), respectively). Of particular importance is also the following transformation formula of $q$-hypergeometric series into $q$-integrals (see [13], p. 26, 1.4 (iii)):

$$
\begin{aligned}
{ }_{r+1} \phi_{r}\left(\begin{array}{c}
a_{1}, \ldots, a_{r+1} \\
b_{1}, \ldots, b_{r}
\end{array} q, q^{z}\right)= & \frac{\left(a_{1}, \ldots, a_{r+1} ; q\right)_{\infty}}{(1-q)\left(q, b_{1}, \ldots, b_{r} ; q\right)_{\infty}} \\
& \times \int_{0}^{1} s^{z-1} \frac{\left(q s, b_{1} s, \ldots, b_{r} s ; q\right)_{\infty}}{\left(a_{1} s, \ldots, a_{r+1} s ; q\right)_{\infty}} d_{q} s .
\end{aligned}
$$

Using these facts we can study the case of no-synchronization (i.e., independent abandonments) that has been investigated by [3]. The following theorem corresponds to their results for the $M / M / 1$ type model (see their Sect. 2, in particular their equations (2.9), (2.8) and (2.3)).

Theorem 3.5 In case $q \rightarrow 1^{-}$and $\zeta(1-q)=\zeta^{*}$ fixed, we have

$$
\begin{aligned}
\pi^{(1)}(0,0) & =\frac{A^{*}}{\zeta^{*}} \int_{0}^{1}(1-s)^{\frac{\gamma}{\zeta^{*}}-1} e^{-\frac{\lambda}{\zeta^{*}} s} d s \\
\Pi_{0}^{(1)}(z) & =\frac{A^{*}}{\zeta^{*}} e^{\frac{\lambda}{\zeta^{*}} z}(1-z)^{-\frac{\gamma}{\zeta *}} \int_{z}^{1}(1-s)^{\frac{\gamma}{\zeta^{*}}-1} e^{-\frac{\lambda}{\zeta^{*}} s} d s \\
\Pi_{1}^{(1)}(z) & =-\frac{A^{*} z}{\lambda z+\mu z-\lambda z^{2}-\mu}+\frac{\gamma z}{\lambda z+\mu z-\lambda z^{2}-\mu} \Pi_{0}^{(1)}(z),
\end{aligned}
$$

where

$$
A^{*}=\frac{\gamma(\mu-\lambda)\left(\gamma+\zeta^{*}\right)}{\mu \gamma+(\mu-\lambda) \zeta^{*}} .
$$

Proof Using (3.44) we express (3.18) as a $q$-integral and we obtain that

$$
\begin{aligned}
\Pi_{0}(z) & =\frac{A}{\gamma+\zeta} \frac{(q ; q)_{\infty}}{\left(-\frac{\lambda(1-z)}{\gamma+\zeta}, \frac{\zeta}{\gamma+\zeta} ; q\right)_{\infty}}{ }_{2} \phi_{1}\left(-\frac{\lambda(1-z)}{\gamma+\zeta}, \frac{\zeta}{\gamma+\zeta} ; 0 ; q, q\right) \\
& =\frac{A}{(\gamma+\zeta)(1-q)} \int_{0}^{1} \frac{(q s ; q)_{\infty}}{\left(-\frac{\lambda(1-z) s}{\gamma+\zeta}, \frac{\zeta s}{\gamma+\zeta} ; q\right)_{\infty}} d_{q} s
\end{aligned}
$$


Taking the limit as $q \rightarrow 1^{-}$and using (3.41)-(3.43) yields

$$
\lim _{q \rightarrow 1^{-}} \Pi_{0}(z)=\frac{A^{*}}{\zeta^{*}} \int_{0}^{1}(1-s)^{\frac{\gamma}{\zeta^{*}}-1} e^{-\frac{\lambda}{\zeta^{*}}(1-z) s} d s,
$$

where $A^{*}=\lim _{q \rightarrow 1^{-}} A$. After a change of variable in (3.50) we arrive at (3.46) which is (2.8) in Altman and Yechiali [3]. Equations (3.45) and (3.47) are now obvious by taking limits as $q \rightarrow 1^{-}$in (3.16) and (3.19).

For the case of full synchronization we have the following theorem.

Theorem 3.6 In case $q \rightarrow 0^{+}$and $\zeta(1-q)=\zeta^{*}$ fixed, we have

$$
\begin{aligned}
& \pi^{(2)}(0,0)=\frac{A^{*}\left(\gamma+\zeta^{*}\right)}{\gamma\left(\gamma+\zeta^{*}+\lambda\right)}, \\
& \pi^{(2)}(n, 0)=\left(\frac{\lambda}{\gamma+\zeta^{*}+\lambda}\right)^{n} \pi^{(2)}(0,0), \quad n \geq 1, \\
& \pi^{(2)}(n, 1)= \begin{cases}\frac{A^{*}}{\gamma+\zeta^{*}+\lambda-\mu}\left[\left(\frac{\lambda}{\mu}\right)^{n}-\left(\frac{\lambda}{\gamma+\zeta^{*}+\lambda}\right)^{n}\right], & n \geq 1, \mu \neq \gamma+\zeta^{*}+\lambda, \\
n \frac{A^{*}}{\mu}\left(\frac{\lambda}{\mu}\right)^{n}, & n \geq 1, \mu=\gamma+\zeta^{*}+\lambda,\end{cases}
\end{aligned}
$$

where

$$
A^{*}=\frac{\gamma(\mu-\lambda)\left(\gamma+\zeta^{*}\right)}{\mu \gamma+(\mu-\lambda) \zeta^{*}}
$$

Proof We take the limit as $q \rightarrow 0^{+}$in (3.18). This yields

$$
\Pi_{0}^{(2)}(z)=\frac{A^{*}\left(\gamma+\zeta^{*}\right)}{\gamma\left(\gamma+\zeta^{*}+\lambda(1-z)\right)},
$$

where $A^{*}$ is given by (3.54). By expanding (3.55) in power series of $z$ we obtain easily (3.51) and (3.52). Taking $q \rightarrow 0^{+}$in (3.19) implies, after some simplifications, that

$$
\Pi_{1}^{(2)}(z)=\frac{A^{*} \lambda z}{\mu\left(\gamma+\zeta^{*}+\lambda\right)\left(1-\frac{\lambda}{\mu} z\right)\left(1-\frac{\lambda}{\gamma+\zeta^{*}+\lambda} z\right)}
$$

By analyzing $\left(\left(1-\frac{\lambda}{\mu} z\right)\left(1-\frac{\lambda}{\gamma+\zeta^{*}+\lambda} z\right)\right)^{-1}$ in partial fractions for the two cases $\mu \neq \gamma+\zeta^{*}+\lambda$ and $\mu=\gamma+\zeta^{*}+\lambda$, and expanding in power series of $z$ we obtain (3.53).

\section{Non-Markovian models: mean value analysis}

We now assume the general framework introduced in Sect. 2, i.e., the service and vacation times are both generally distributed. The analysis is similar for the two models, 
so we treat them simultaneously up to the point where the abandonment mechanism enters.

We suppose that the system is in equilibrium and we consider as before the random variables $L$ representing the number of customers in the system and $S$ representing the sojourn time of a customer. Let also $L_{i}$ be the conditional number of customers in the system, given that the server is in state $i, i=0,1$. Further we denote by $p_{i}$ the probability (or fraction of time) that the server is in state $i, i=0,1$. Let us consider a tagged arriving customer. Then, by PASTA, the probability that this customer finds the server in state $i$ is $p_{i}$ and in this case, he finds on average $E\left(L_{i}\right)$ customers in front of him.

If he finds the server providing service, then his mean sojourn time is equal to the residual service time of the customer in service plus the service times of all customers waiting in the queue plus his own service time. Hence, his mean sojourn time is $E\left(B_{\mathrm{e}}\right)+\left(E\left(L_{1}\right)-1\right) E(B)+E(B)$.

If he finds the server on vacation, then he has to wait for the vacation time to expire before servicing starts and he may decide to abandon at one of the abandonment opportunities (there is just one in case of the UAE model, but possibly many in case of the MAE model). Let $V^{*}$ be his time in the system till the end of the vacation. If the tagged customer is still in the system just after the end of the vacation, then his sojourn time after the return of the server depends on the number of customers (still) in front of him. Define $\pi$ as the probability that, just after the end of the vacation period, the tagged customer is still in the system and, accordingly, define $\pi^{*}$ as the probability that the tagged customer and a customer, who was already present at his arrival, are both still in the system. Then we have that the mean number of customers in front of him just after the end of the vacation period is $\pi^{*} E\left(L_{0}\right)$. Hence

$$
E(S)=p_{1}\left(E\left(B_{\mathrm{e}}\right)+E\left(L_{1}\right) E(B)\right)+p_{0}\left(E\left(V^{*}\right)+\pi^{*} E\left(L_{0}\right) E(B)+\pi E(B)\right) .
$$

Further, Little's law states that

$$
E(L)=\lambda E(S)
$$

Also, if we would act as if the customers arriving during a vacation are waiting in a "vacation area" and transferred to the queue as soon as the server returns, then application of Little's law to the vacation area yields

$$
E\left(L_{0}\right)=\lambda E\left(V^{*}\right)
$$

The unconditional $E(L)$ is related to the conditional ones as

$$
E(L)=p_{0} E\left(L_{0}\right)+p_{1} E\left(L_{1}\right) .
$$

Conservation of work gives

$$
p_{1}=\left(\lambda p_{0} \pi+\lambda p_{1}\right) E(B),
$$

and

$$
p_{0}+p_{1}=1 \text {. }
$$


If we determine $\pi$, then (4.5)-(4.6) yield immediately the probabilities $p_{0}$ and $p_{1}$. If we also determine $\pi^{*}$ and $E\left(V^{*}\right)$, then (4.1)-(4.4) suffice for the computation of the unknown mean values $E(L), E\left(L_{0}\right), E\left(L_{1}\right)$ and $E(S)$. The computations of $\pi$, $\pi^{*}$ and $E\left(V^{*}\right)$ depend on the specific abandonment mechanism, so we treat the UAE and the MAE model separately.

\subsection{Mean value analysis of the UAE model}

In the UAE model a customer that arrives when the server is on vacation has a unique opportunity to abandon the system at the end of the vacation. Therefore he will stay in the system for the residual vacation time and then he will decide whether to leave or not, so $E\left(V^{*}\right)=E\left(V_{\mathrm{e}}\right)$. The probability $\pi$ is clearly equal to $q$, while $\pi^{*}$ is equal to $q^{2}$. Solution of (4.1)-(4.6) yields:

Theorem 4.1 The mean sojourn time is given by

$$
E(S)=\frac{1}{1-\rho p}\left(E\left(V_{\mathrm{e}}\right)+\left(q^{2}-1\right) \rho E\left(V_{\mathrm{e}}\right)\right)+\frac{q}{1-\rho p}\left(\frac{\rho E\left(B_{\mathrm{e}}\right)}{1-\rho}+E(B)\right),
$$

and the fractions of time the server is inactive and active are, respectively,

$$
p_{0}=\frac{1-\rho}{1-\rho p}, \quad p_{1}=\frac{\rho q}{1-\rho p} .
$$

In particular, in the Markovian case we have the following corollary.

Theorem 4.2 The mean sojourn time is given by

$$
E(S)=\frac{1}{1-\rho p}\left(\frac{1}{\gamma}+\left(q^{2}-1\right) \frac{\rho}{\gamma}\right)+\frac{q}{1-\rho p} \cdot \frac{1}{\mu(1-\rho)},
$$

and the fractions of time the server is inactive and active, respectively, are given by (4.7).

\subsection{Mean value analysis of the MAE model}

In the MAE model a customer that arrives when the server is on vacation may have several opportunities to abandon the system. The computation of $\pi, \pi^{*}$ and $E\left(V^{*}\right)$ is not immediate as in case of the UAE model. However, we observe that, if the tagged customer arrives during a vacation, then the time till abandonment is exponential with rate $\zeta p$. By denoting the time till abandonment by $T$, we can write

$$
V^{*}=\min \left(V_{\mathrm{e}}, T\right) \quad \text { and } \quad \pi=P\left(V_{\mathrm{e}}<T\right) .
$$

Hence, by conditioning on the length of $V_{\mathrm{e}}$,

$$
\pi=\int_{0}^{\infty} P(t<T) d V_{\mathrm{e}}(t)
$$




$$
\begin{aligned}
& =\int_{0}^{\infty} e^{-\zeta p t} d V_{\mathrm{e}}(t) \\
& =\tilde{V}_{\mathrm{e}}(\zeta p)
\end{aligned}
$$

and

$$
\begin{aligned}
E\left(V^{*}\right) & =E\left(V_{\mathrm{e}}+T\right)-E\left(\max \left(V_{\mathrm{e}}, T\right)\right) \\
& =E\left(V_{\mathrm{e}}\right)+\frac{1}{\zeta p}-\left(E\left(V_{\mathrm{e}}\right)+\pi \frac{1}{\zeta p}\right) \\
& =(1-\pi) \frac{1}{\zeta p} .
\end{aligned}
$$

To compute $\pi^{*}$ we condition on the length of $V_{\mathrm{e}}$ and on the number of abandonment epochs during $V_{\mathrm{e}}$,

$$
\begin{aligned}
\pi^{*} & =\int_{0}^{\infty} \sum_{n=0}^{\infty} e^{-\zeta t} \frac{(\zeta t)^{n}}{n !}\left(q^{2}\right)^{n} d P\left(V_{\mathrm{e}} \leq t\right) \\
& =\int_{0}^{\infty} e^{-\zeta t} e^{\zeta t q^{2}} d V_{\mathrm{e}}(t) \\
& =\int_{0}^{\infty} e^{-\zeta\left(1-q^{2}\right) t} d V_{\mathrm{e}}(t) \\
& =\tilde{V}_{\mathrm{e}}\left(\zeta\left(1-q^{2}\right)\right)
\end{aligned}
$$

By solving (4.1)-(4.6), and taking into account (4.8)-(4.10), we finally get:

Theorem 4.3 The mean sojourn time is equal to $E(S)=\frac{1}{1-\rho+\rho \pi}\left(E\left(V^{*}\right)+\left(\pi^{*}-1\right) \rho E\left(V^{*}\right)\right)+\frac{\pi}{1-\rho+\rho \pi}\left(\frac{\rho E\left(B_{\mathrm{e}}\right)}{1-\rho}+E(B)\right)$, where $\pi, \pi^{*}$ and $E\left(V^{*}\right)$ are given by (4.8), (4.10) and (4.9), respectively, and

$$
p_{0}=\frac{1-\rho}{1-\rho+\rho \pi}, \quad p_{1}=\frac{\rho \pi}{1-\rho+\rho \pi} .
$$

In the Markovian case we can compute $\pi, \pi^{*}$ and $E\left[V^{*}\right]$ explicitly. Indeed, we have

$$
\begin{aligned}
\pi & =\frac{\gamma}{\zeta p+\gamma}, \\
\pi^{*} & =\frac{\gamma}{\zeta\left(1-q^{2}\right)+\gamma},
\end{aligned}
$$

and

$$
E\left(V^{*}\right)=\frac{1}{\zeta p+\gamma}
$$


Thus, we have the following corollary.

Theorem 4.4 The mean sojourn time is given by

$$
\begin{aligned}
E(S)= & \frac{1}{1-\rho+\rho \pi}\left(\frac{1}{\zeta p+\gamma}+\left(q^{2}-1\right) \frac{\zeta \pi}{\zeta\left(1-q^{2}\right)+\gamma} \cdot \frac{\rho}{\gamma}\right) \\
& +\frac{\pi}{1-\rho+\rho \pi} \cdot \frac{1}{\mu(1-\rho)}
\end{aligned}
$$

where $\pi$ is given by (4.12) and the fractions of time the server is inactive and active, respectively, are given by (4.11).

\section{Non-Markovian models: equilibrium distribution}

The aim of this section is to determine the PGF of the number of customers in the system. By conditioning on the state of the server, we obtain

$$
E\left(z^{L}\right)=p_{0} E\left(z^{L_{0}}\right)+p_{1} E\left(z^{L_{1}}\right) .
$$

To find the PGF of $L_{1}$ we first need the number of customers in the system just after the end of a vacation; denote this number by $L_{v}$. We now proceed as in [12]. Define the primary customers to be the ones just after the start of the busy period and the secondary customers to be the ones who arrive during the busy period. Further, we change the service discipline to non-preemptive LCFS; this does not affect the number of customers in the system. So, after servicing a primary customer, the server will serve any secondary customer until there is none present. Each primary customer generates a standard $M / G / 1$ busy period, at the end of which the server either begins servicing the next primary customer or, if the system is empty, takes a vacation. Let $Q_{p}$ be the number of primary customers waiting for service in the queue (excluding the one possibly in service). If we remove server vacations from the time axis and glue together the service periods, then we readily obtain from the renewal reward theorem (see, e.g., [21]) that the fraction of time the queue contains $n$ primary customers is equal to

$$
P\left(Q_{p}=n\right)=\frac{P\left(L_{v}>n\right)}{E\left(L_{v}\right)}, \quad n \geq 0 .
$$

Hence,

$$
E\left(z^{Q_{p}}\right)=\frac{1}{E\left(L_{v}\right)} \sum_{n=0}^{\infty} P\left(L_{v}>n\right) z^{n}=\frac{1-E\left(z^{L_{v}}\right)}{E\left(L_{v}\right)(1-z)} .
$$

Let $L_{1 \mid M / G / 1}$ denote the conditional number of customers in the corresponding standard $M / G / 1$ with arrival rate $\lambda$ and service time distribution $B(t)$, given that the server is in state 1 (i.e., active); so, according to the Pollaczek-Khinchin formula,

$$
E\left(z^{L_{1 \mid M / G / 1}}\right)=\frac{(1-\rho)}{\rho} \cdot \frac{z(1-\tilde{B}(\lambda(1-z)))}{\tilde{B}(\lambda(1-z))-z} .
$$


Since $L_{1}=L_{1 \mid M / G / 1}+Q_{p}$, where $L_{1 \mid M / G / 1}$ and $Q_{p}$ are independent, we obtain, by (5.2) and (5.3),

$$
\begin{aligned}
E\left(z^{L_{1}}\right) & =E\left(z^{L_{1 \mid M / G / 1}}\right) E\left(z^{Q_{p}}\right) \\
& =\frac{(1-\rho)}{\rho} \cdot \frac{z(1-\tilde{B}(\lambda(1-z)))}{\tilde{B}(\lambda(1-z))-z} \cdot \frac{1-E\left(z^{L_{v}}\right)}{E\left(L_{v}\right)(1-z)} .
\end{aligned}
$$

It is now clear that the determination of the PGF of the number of customers in the system reduces to the computation of $E\left(z^{L_{0}}\right), E\left(z^{L_{v}}\right)$ and $E\left(L_{v}\right)$. These computations depend on the specific abandonment mechanism, so we treat the UAE and the MAE model separately.

\subsection{Equilibrium distribution of the UAE model}

In this case the probabilities $p_{0}$ and $p_{1}$ are given by (4.7). The number of customers during a vacation, $L_{0}$, are exactly the ones who arrived during the age of the vacation, and the age is in distribution the same as the residual vacation. Hence, by conditioning on $V_{\mathrm{e}}=t$, the number of arrivals is Poisson with parameter $\lambda t$, and thus we get

$$
\begin{aligned}
E\left(z^{L_{0}}\right) & =\int_{0}^{\infty} e^{-\lambda t(1-z)} d V_{\mathrm{e}}(t) \\
& =\tilde{V}_{\mathrm{e}}(\lambda(1-z))
\end{aligned}
$$

The number of arrivals during a vacation of length $t$, who decide to stay at the end of the vacation, is Poisson with parameter $q \lambda t$. Hence, the PGF of the number of customers in the system, just after the end of the vacation, is

$$
E\left(z^{L_{v}}\right)=\tilde{V}(q \lambda(1-z))
$$

We can now combine (5.1), (5.4), (5.5), (5.6) and (2.1) to obtain the PGF of the number of customers in the system. We have the following theorem.

Theorem 5.1 The PGF of the number of customers in the system is given by

$$
E\left(z^{L}\right)=\frac{1-\rho}{1-\rho p} \tilde{V}_{\mathrm{e}}(\lambda(1-z))+\frac{q(1-\rho)}{1-\rho p} \frac{z(1-\tilde{B}(\lambda(1-z)))}{\tilde{B}(\lambda(1-z))-z} \tilde{V}_{\mathrm{e}}(q \lambda(1-z)) .
$$

Remark 5.1 In case $q=1-p=1$, the equation above reduces to the well-known Fuhrmann-Cooper decomposition for the number of customers in the $M / G / 1$ with server vacations (and no abandonments),

$$
E\left(z^{L}\right)=V_{\mathrm{e}}(\lambda(1-z)) E\left(z^{L_{M / G / 1}}\right)
$$

where $L_{M / G / 1}$ is the (unconditional) number of customers in the corresponding standard $M / G / 1$. 


\subsection{Equilibrium distribution of the MAE model}

In this case the probabilities $p_{0}$ and $p_{1}$ are given by (4.11). We need also to obtain $E\left(z^{L_{0}}\right), E\left(z^{L_{v}}\right)$ and $E\left(L_{v}\right)$. We start with the latter. Conditioning on the event that $V=t$, the number of abandonment epochs is Poisson with parameter $\zeta t$. Given the number of abandonment epochs is $n(>0)$, the event times $\left(s_{1}, s_{2}, \ldots, s_{n}\right)$ of these epochs will be distributed as the order statistics $\left(U_{1: n}, U_{2: n}, \ldots, U_{n: n}\right)$ of a random sample $\left(U_{1}, U_{2}, \ldots, U_{n}\right)$ from the uniform distribution in $(0, t]$. The number of arrivals in each of the intervals $\left(0, s_{1}\right],\left(s_{1}, s_{2}\right], \ldots,\left(s_{n-1}, s_{n}\right],\left(s_{n}, t\right]$ are Poisson with parameters $\lambda s_{1}, \lambda\left(s_{2}-s_{1}\right), \ldots, \lambda\left(s_{n}-s_{n-1}\right), \lambda\left(t-s_{n}\right)$ respectively. Moreover, the individuals that arrive during these intervals will remain till time $t$ with probabilities $q^{n}, q^{n-1}, \ldots, q, 1$ respectively. Since the sum of Poisson random variables is again Poisson, we can conclude that the number of customers at the end of the vacation is Poisson with parameter

$$
\begin{aligned}
\Lambda\left(t, n, s_{1}, \ldots, s_{n}\right) & =\lambda s_{1} q^{n}+\lambda\left(s_{2}-s_{1}\right) q^{n-1}+\cdots+\lambda\left(s_{n}-s_{n-1}\right) q+\lambda\left(t-s_{n}\right) \\
& =-\lambda q^{n-1}(1-q) s_{1}-\lambda q^{n-2}(1-q) s_{2}-\cdots-\lambda(1-q) s_{n}+\lambda t,
\end{aligned}
$$

valid for $n>0$, and if $n=0$, this number is Poisson with parameter $\lambda t$. Hence,

$$
\begin{aligned}
E\left(z^{L_{v}} \mid V=t\right)= & e^{-\zeta t} e^{-\lambda t(1-z)}+\sum_{n=1}^{\infty} \int_{0}^{t} \int_{s_{1}}^{t} \cdots \int_{s_{n-1}}^{t} e^{-\zeta t} \frac{(\zeta t)^{n}}{n !} \\
& \times e^{-\Lambda\left(t, n, s_{1}, \ldots, s_{n}\right)(1-z)} \frac{n !}{t^{n}} d s_{n} \cdots d s_{1} .
\end{aligned}
$$

To put $E\left(z^{L_{v}} \mid V=t\right)$ in a more compact form, we use the auxiliary identity

$$
\begin{aligned}
I_{n}\left(t, n, \alpha_{1}, \alpha_{2}, \ldots, \alpha_{n}\right)= & \int_{0}^{t} \int_{s_{1}}^{t} \cdots \int_{s_{n-1}}^{t} e^{\alpha_{1} s_{1}+\alpha_{2} s_{2}+\cdots+\alpha_{n} s_{n}} d s_{n} \cdots d s_{2} d s_{1} \\
= & \sum_{k=1}^{n+1}(-1)^{k+1} e^{t \sum_{i=k}^{n} \alpha_{i}} \\
& \times \frac{1}{\prod_{i=0}^{n-k} \sum_{j=k}^{k+i} \alpha_{j} \cdot \prod_{i=1}^{k-1} \sum_{j=k-i}^{k-1} \alpha_{j}},
\end{aligned}
$$

which can be easily established by induction. In order to use (5.9) to simplify (5.8), we substitute

$$
\alpha_{j}=\lambda(1-q)(1-z) q^{n-j}, \quad j=1,2, \ldots, n
$$

into (5.9), yielding (after some algebra)

$$
\begin{aligned}
I_{n}\left(t, n, \alpha_{1}, \alpha_{2}, \ldots, \alpha_{n}\right)= & \frac{e^{t \lambda(1-z)}}{[\lambda(1-z)]^{n}} \sum_{k=0}^{n}(-1)^{k} e^{-t \lambda(1-z) q^{n-k}} \\
& \cdot \frac{1}{(q ; q)_{k}(q ; q)_{n-k} q^{\left(\frac{n-k}{2}\right)+(n-k) k}} .
\end{aligned}
$$


Using (5.8), (5.9) and (5.11) we obtain

$$
\begin{aligned}
E\left(z^{L_{v}} \mid V=t\right)= & e^{-(\zeta+\lambda(1-z)) t} \sum_{n=0}^{\infty} \zeta^{n} \sum_{k=0}^{n} \frac{e^{t \lambda(1-z)}}{(\lambda(1-z))^{n}} \frac{1}{(q ; q)_{k}(q ; q)_{n-k}} \\
& \times(-1)^{k} e^{-t \lambda(1-z) q^{n-k}} q^{-(n+k-1)(n-k) / 2} \\
= & \frac{e^{-\zeta t}}{\left(-\frac{\zeta}{\lambda(1-z)} ; q\right)_{\infty}} \sum_{n=0}^{\infty} \frac{q^{n} e^{-t \lambda(1-z) q^{n}}}{(q ; q)_{n}\left(-\frac{\lambda(1-z) q}{\zeta} ; q\right)_{n}}
\end{aligned}
$$

Hence, after unconditioning, we conclude that the PGF of $L_{v}$ assumes the form

$$
\begin{aligned}
E\left(z^{L_{v}}\right) & =\int_{0}^{\infty} E\left(z^{L_{v}} \mid V=t\right) d V(t) \\
& =\int_{0}^{\infty} \frac{e^{-\zeta t}}{\left(-\frac{\zeta}{\lambda(1-z)} ; q\right)_{\infty}} \sum_{n=0}^{\infty} \frac{q^{n}}{(q ; q)_{n}} e^{-t \lambda(1-z) q^{n}} \frac{1}{\left(-\frac{\lambda(1-z) q}{\zeta} ; q\right)_{n}} d V(t) \\
& =\frac{1}{\left(-\frac{\zeta}{\lambda(1-z)} ; q\right)_{\infty}} \sum_{n=0}^{\infty} \frac{q^{n} \tilde{V}\left(\zeta+\lambda(1-z) q^{n}\right)}{(q ; q)_{n}\left(-\frac{\lambda(1-z) q}{\zeta} ; q\right)_{n}}
\end{aligned}
$$

Moreover,

$$
\begin{aligned}
E\left(L_{v} \mid V=t\right)= & e^{-\zeta t} \lambda t+\sum_{n=1}^{\infty} \int_{0}^{t} \int_{s_{1}}^{t} \cdots \int_{s_{n-1}}^{t} e^{-\zeta t} \frac{(\zeta t)^{n}}{n !} \\
& \times \Lambda\left(t, n, s_{1}, \ldots, s_{n}\right) \frac{n !}{t^{n}} d s_{n} \cdots d s_{1} .
\end{aligned}
$$

To put $E\left(L_{v} \mid V=t\right)$ in a more compact form we use the auxiliary identity

$$
\begin{aligned}
J_{n}\left(t, n, \alpha_{1}, \alpha_{2}, \ldots, \alpha_{n}\right)= & \int_{0}^{t} \int_{s_{1}}^{t} \cdots \int_{s_{n-1}}^{t}\left[\alpha_{0}+\alpha_{1} s_{1}+\alpha_{2} s_{2}+\cdots+\alpha_{n} s_{n}\right] \\
& \times d s_{n} \cdots d s_{2} d s_{1} \\
= & \alpha_{0} \frac{t^{n}}{n !}+\sum_{k=1}^{n} \alpha_{k} \frac{k t^{n+1}}{(n+1) !}
\end{aligned}
$$

which can be easily established by induction. In order to use (5.15) to simplify (5.14) we substitute

$$
\begin{aligned}
& \alpha_{0}=\lambda t \\
& \alpha_{j}=-\lambda(1-q) q^{n-j}, \quad j=1,2, \ldots, n
\end{aligned}
$$

into (5.15), yielding

$$
J_{n}\left(t, n, \alpha_{1}, \alpha_{2}, \ldots, \alpha_{n}\right)=\lambda \frac{t^{n+1}}{(n+1) !} \frac{1-q^{n+1}}{1-q} .
$$


Using (5.14) and (5.16) we obtain

$$
E\left(L_{v} \mid V=t\right)=\frac{\lambda}{\zeta p}\left(1-e^{-\zeta p t}\right) .
$$

Note that, after unconditioning, the mean value of $L_{v}$ assumes the form

$$
E\left(L_{v}\right)=\frac{\lambda}{\zeta p}(1-\tilde{V}(\zeta p))
$$

To determine the PGF of the number of customers during a vacation, we can copy the approach above, where the vacation $V$ should be replaced by its age $V_{\mathrm{e}}$. This leads to

$$
\begin{aligned}
E\left(z^{L_{0}}\right) & =\int_{0}^{\infty} E\left(z^{L_{0}} \mid V_{\mathrm{e}}=t\right) d V_{\mathrm{e}}(t) \\
& =\int_{0}^{\infty} \frac{e^{-\zeta t}}{\left(-\frac{\zeta}{\lambda(1-z)} ; q\right)_{\infty}} \sum_{n=0}^{\infty} \frac{q^{n}}{(q ; q)_{n}} e^{-t \lambda(1-z) q^{n}} \frac{1}{\left(-\frac{\lambda(1-z) q}{\zeta} ; q\right)_{n}} d V_{\mathrm{e}}(t) \\
& =\frac{1}{\left(-\frac{\zeta}{\lambda(1-z)} ; q\right)_{\infty}} \sum_{n=0}^{\infty} \frac{q^{n} \tilde{V}_{\mathrm{e}}\left(\zeta+\lambda(1-z) q^{n}\right)}{(q ; q)_{n}\left(-\frac{\lambda(1-z) q}{\zeta} ; q\right)_{n}}
\end{aligned}
$$

Based on the PGFs of $L_{0}, L_{v}$ and the mean value $E\left(L_{v}\right)$, we can now use (5.1), (5.4), (5.19) and (5.13) to obtain the PGF of the number of customers in the system. Therefore, we immediately obtain the following result.

Theorem 5.2 The PGF of the number of customers in the system is given by

$$
\begin{aligned}
E\left(z^{L}\right)= & \frac{1-\rho}{1-\rho+\rho \pi} E\left(z^{L_{0}}\right) \\
& +\frac{\pi(1-\rho)}{1-\rho+\rho \pi} \frac{z(1-\tilde{B}(\lambda(1-z)))}{\tilde{B}(\lambda(1-z))-z} \frac{1-E\left(z^{L_{v}}\right)}{E\left(L_{v}\right)(1-z)},
\end{aligned}
$$

where $\pi, E\left(z^{L_{0}}\right), E\left(z^{L_{v}}\right)$ and $E\left(L_{v}\right)$ are given by (4.8), (5.19), (5.13) and (5.18), respectively.

Remark 5.2 As the arrival rate $\zeta$ of the abandonment epochs tends to zero (i.e., no abandonments), then (5.20) again reduces to the standard decomposition (5.7). To show this, we first use the identity

$$
(a ; q)_{n}=\left(a^{-1} q^{1-n} ; q\right)_{n}(-a)^{n} q^{\left(\begin{array}{c}
n \\
2
\end{array}\right)}
$$

to rewrite (5.13) in the form

$$
E\left(z^{L_{v}}\right)=\sum_{n=0}^{\infty} \frac{\zeta^{n}}{(\lambda(1-z))^{n}} \frac{\tilde{V}\left(\zeta+\lambda(1-z) q^{n}\right)}{(q ; q)_{n}\left(-\frac{\zeta q^{-n}}{\lambda(1-z)} ; q\right)_{\infty} q^{\left(\begin{array}{c}
n \\
2
\end{array}\right)}}
$$


For $\zeta=0$ this equation reduces to $E\left(z^{L_{v}}\right)=\tilde{V}(\lambda(1-z))$. Similarly, it can be shown that $E\left(z^{L_{0}}\right)=\tilde{V}_{\mathrm{e}}(\lambda(1-z))$ for $\zeta=0$. Substitution of these expressions into (5.20) yields (5.7).

\section{Numerical results}

This section is devoted to several numerical results that shed further light on the effect of the various model parameters and distributions on the system performance. To this end we perform several numerical experiments by keeping all but one parameter fixed and study the mean number of customers in the system as a function of the varying parameter. The effect of $\lambda, E(B)$ or $E(V)$ on the mean number of customers in the system, $E(L)$, appears to be what is normally expected, i.e., $E(L)$ is increasing in $\lambda$, $E(B)$ and $E(V)$. Much more interesting is the effect of the abandonment probability $p$ on $E(L)$. In the numerical scenarios presented below we assume that $B$ is exponentially distributed with $E(B)=1$, and to show the effect of the dispersion of the vacation times, we consider Erlang, exponential and hyper-exponential vacation time distributions.

Another interesting question is the speed of convergence to the fluid limit. To this end we plot a graph of $E\left(L^{m} / m\right)$, for the sequence of models with $\lambda_{m}=m \lambda$ and $\mu_{m}=m \mu$ (while keeping all other model parameters fixed) to illustrate the rate of convergence to $E(\bar{L})$.

We present the results for the UAE and the MAE model separately, as they are qualitatively different.

\subsection{Numerical results for the UAE model}

For the UAE model, we plot the graph of $E(L)$ as a function of $p$, while keeping all other parameters fixed. We consider two cases regarding the mean vacation time $E(V)$ that correspond to Figs. 5 and 6 . Figure 5, where $E(V)=1$, corresponds to 'small' vacation times, while Fig. 6 , where $E(V)=10$, corresponds to 'large' vacation times. Moreover, in every figure we plot three curves, each corresponding to a different vacation time distribution (Erlang, exponential and hyper-exponential). We observe that $E(L)$ increases as the coefficient of variation of the vacation time, $c_{V}$, increases, while keeping $E(V)$ fixed. This agrees with the usual observation that congestion increases with variability.

In Fig. 5 the mean number of customers $E(L)$ is decreasing with respect to the abandonment probability $p$. This agrees with our intuition that 'the greater the abandonment probability, the less the congestion of the system.' However, Fig. 6 shows that this is not always the case, i.e., the mean number of customers $E(L)$ may exhibit non-monotonic behavior with respect to the abandonment probability $p$. Thus, an increase in the abandonment probability may lead to an increase of the mean number of customers in the system!

This finding may be intuitively justified as follows. Indeed, for large vacation times, the mean number of customers $E(L)$ depends primarily on what happens when the server is on vacation. However, the abandonment probability $p$ does not influence the mean number of customers given that the server is on vacation, since the unique 
Fig. $5 E(L)$ versus $p$

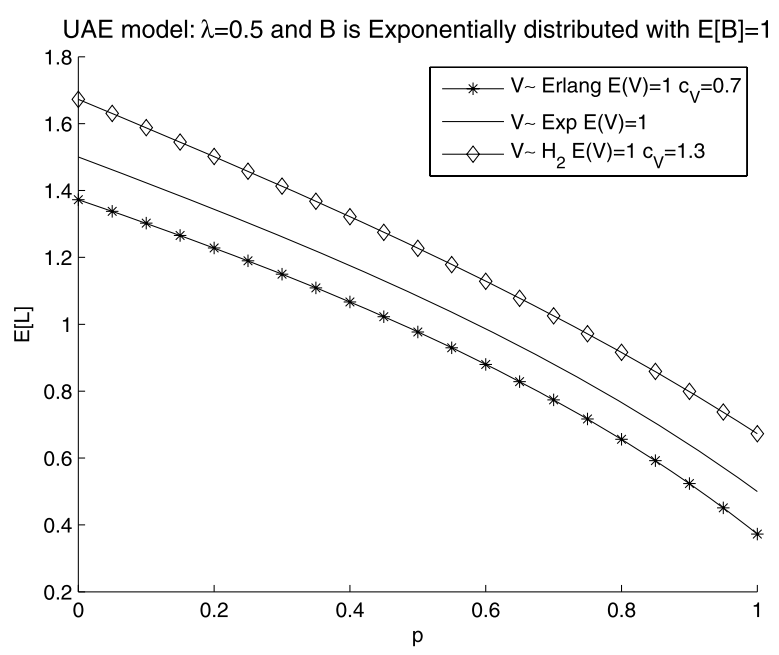

Fig. $6 E(L)$ versus $p$

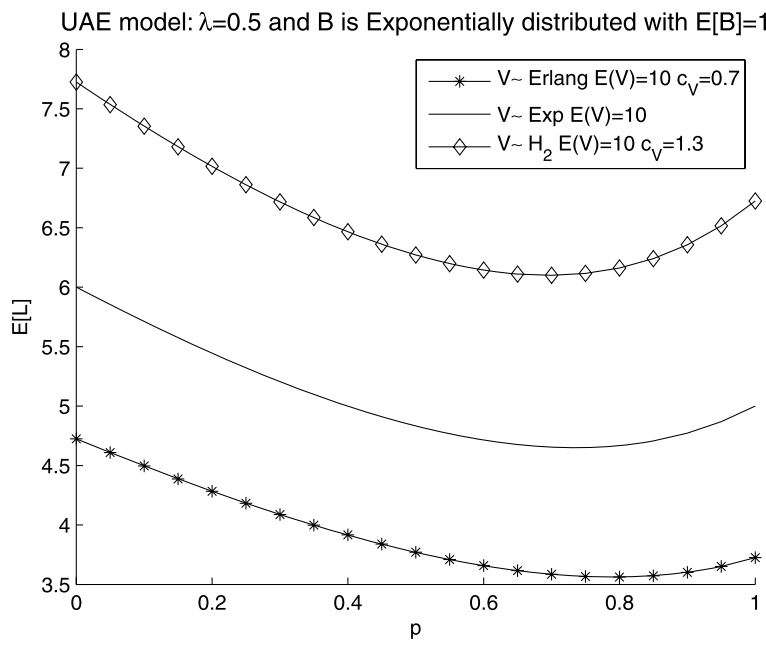

abandonment epoch occurs at the end of the vacation. Moreover, a large number of customers will accumulate during a large vacation time. Thus, in this case, a high abandonment probability $p$ implies that the busy period will start with just a few customers, so the next vacation period which is responsible for the accumulation of many customers in the system will start soon. So, in this case, an increase of the abandonment probability implies an increase of the congestion of the system.

In Fig. 7 we present the convergence of the mean number of customers $E\left(L^{m} / m\right)$ for the sequences of models with arrival rates $\lambda_{m}=m \lambda$, service rates $\mu_{m}=m \mu$ and constant vacation rates $\gamma_{m}=\gamma$ to the mean of the fluid model $E(\bar{L})$.

\subsection{Numerical results for the MAE model}

For the MAE model the most important numerical finding concerns the effect of synchronization. Figures 8-10 demonstrate the effect of the level of synchronization on 
Fig. 7 Convergence of $E\left(L^{m} / m\right)$ to $E(\bar{L})$ of the fluid model
UAE model: $\lambda=0.5, B$ is Exponentially distributed with $E[B]=1$, $V$ is Exponentially distributed with $E[V]=1$ $p=0.5$

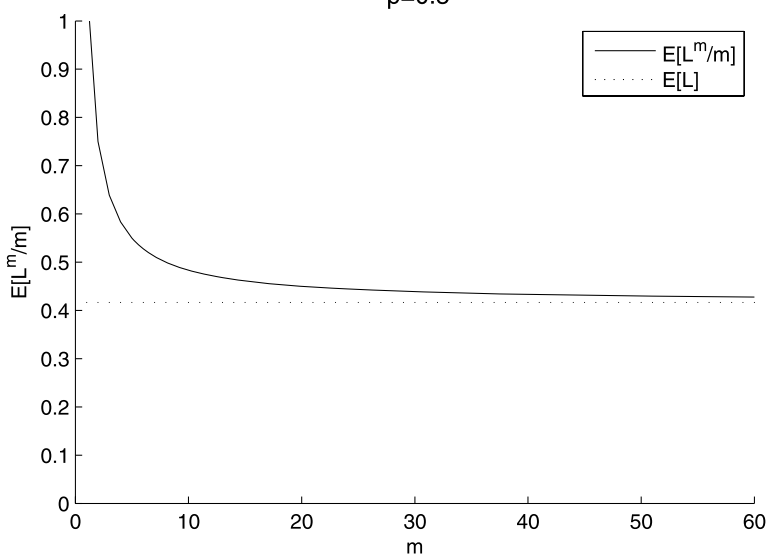

Fig. $8 E(L)$ versus $p, \zeta^{*}$ fixed

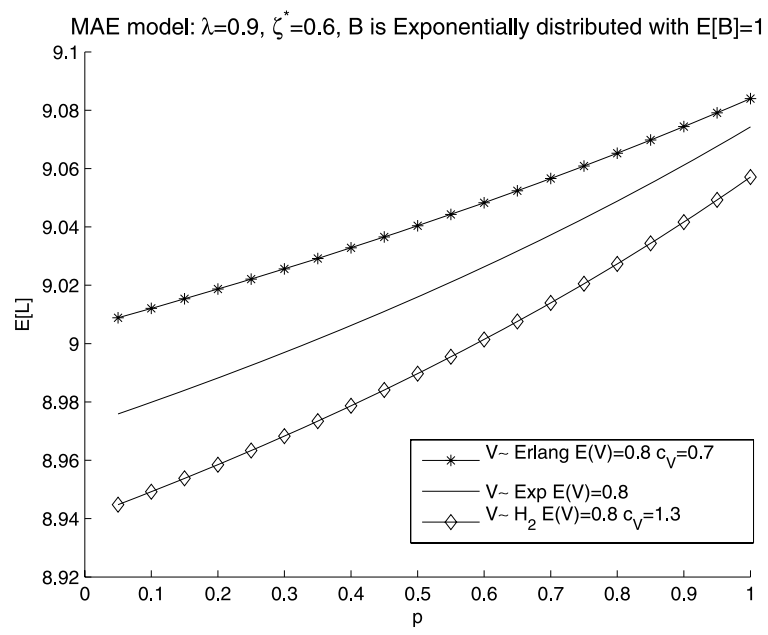

$E(L)$. In these figures, the arrival rate $\lambda$, the effective abandonment rate per customer $\zeta^{*}=\zeta p$ and the mean service time $E(B)$ are kept fixed, i.e., $\lambda=0.9, \zeta^{*}=0.6$ and $E(B)=1$, while $\zeta$ is considered as a function of $p$. In every graph we consider a different value for $E(V)$, i.e. $E(V)=0.8, E(V)=0.4$ and $E(V)=0.25$ in Figs. 8, 9 and 10 , respectively. In each graph we consider three different cases for the distribution of $V(x)$ being Erlang, exponential or hyper-exponential with coefficients of variation $c_{V}=0.7, c_{V}=1$ and $c_{V}=1.3$. For all cases we observe that $E(L)$ is an increasing convex function of $p$, i.e., the more the synchronization the more the congestion. Indeed, this finding has also been observed in other systems with synchronization characteristics (see, e.g., [11]). On the other hand, the effect of the variance of the vacation times is not clear. Figure 8 shows that for systems with large mean vacation times, the more variable the vacation times, the less congested the system. In contrast, Fig. 10 shows that for systems with small mean vacation times, the mean number of customers increases with the variation of the vacation times. Moreover, for 
Fig. $9 E(L)$ versus $p, \zeta^{*}$ fixed

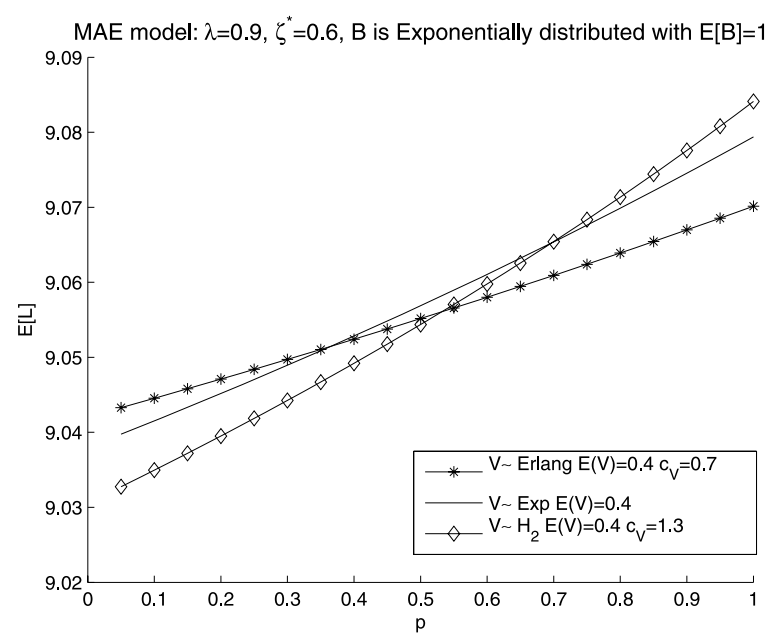

Fig. $10 E(L)$ versus $p, \zeta^{*}$ fixed

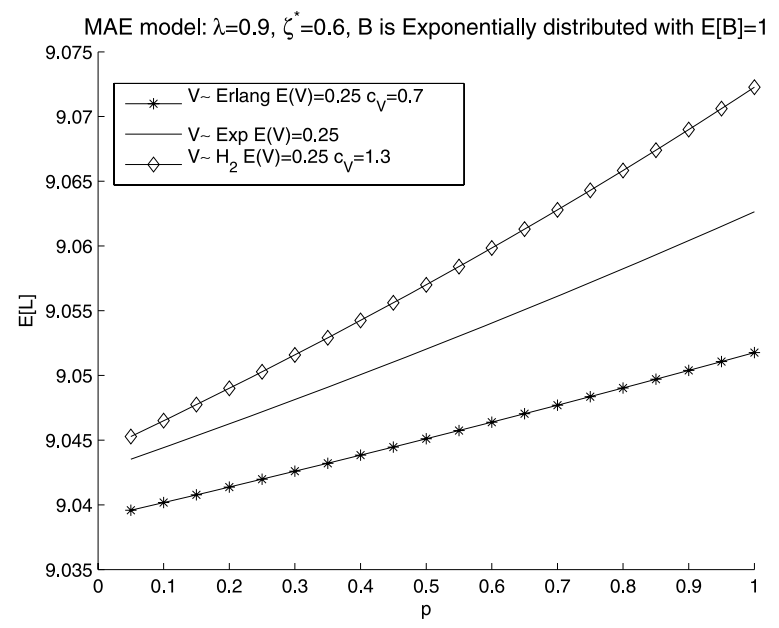

moderate mean vacation times, the situation is mixed (see Fig. 9). In summary, as the mean vacation time increases, the effect of the vacation variability on the congestion of the system turns from negative to positive!

This finding can be intuitively justified as follows. For large vacation times, the number of customers at the end of a vacation period tends to a fixed number (see the formula for $E\left(L_{v}\right)$ ). Hence big and very big vacation times are then followed by busy periods that start from practically the same mean number of customers. So a more irregular (more variable) distribution for the vacation times leaves the possibility for some small vacation times and then the subsequent busy periods will start with significantly fewer customers. Thus the more variable the vacation time distribution, the more likely there exist busy periods starting with few customers. In contrast, vacation times with a large mean but a small variance imply that almost all busy periods start with the same big number of customers. Hence, for large mean vacation times, the variability of the vacation time has a positive effect on the performance of the system, 
in the sense that it reduces congestion. For small mean vacation times this effect is reversed.

In Fig. 11 we present the graph of $E(L)$ with respect to $p$. In this figure, the arrival rate $\lambda$, the mean vacation time $E(V)$, the abandonment rate $\zeta$ and the mean service time $E(B)$ are kept fixed, $\lambda=0.5, E(V)=1, \zeta=1.5$ and $E(B)=1$. The function is decreasing convex as $p$ varies from 0 to 1 . In this case, the effect of the variance of vacation time is that the more regular the distribution the milder the effect of the abandonment probability $p$ on the mean number of customers in the system.

Finally, in Fig. 12 we present the convergence of the mean number of customers $E\left(L^{m} / m\right)$ for the sequences of models with arrival rates $\lambda_{m}=m \lambda$, service rates $\mu_{m}=m \mu$, constant vacation rates $\gamma_{m}=\gamma$ and constant abandonment rates $\zeta_{m}=\zeta$ to the mean of the fluid model $E(\bar{L})$.

Fig. $11 E(L)$ versus $p, \zeta$ fixed

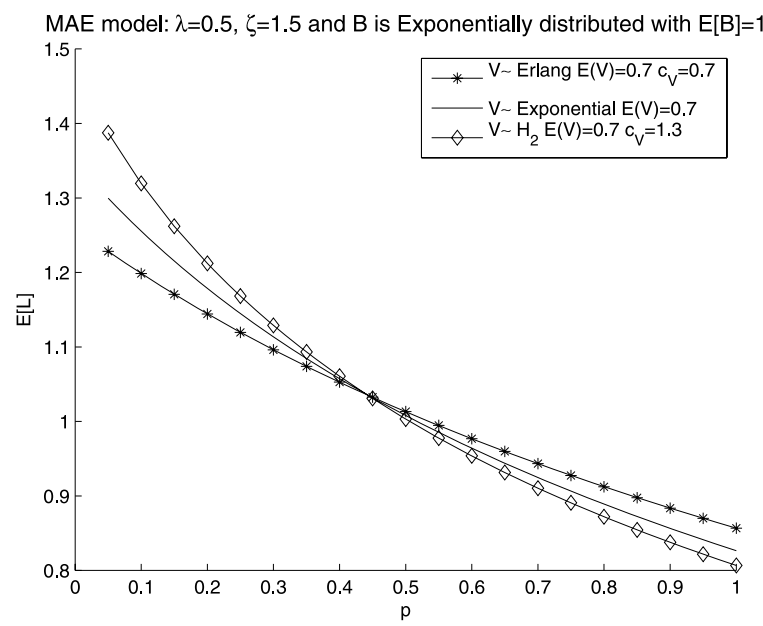

Fig. 12 Convergence of $E\left(L^{m} / m\right)$ to $E(\bar{L})$ of the fluid model
MAE model: $\lambda=0.5, B$ is Exponentially distributed with $E[B]=1$, $V$ is Exponentially distributed with $E[V]=2$ $\zeta^{*}=1 p=0.6$

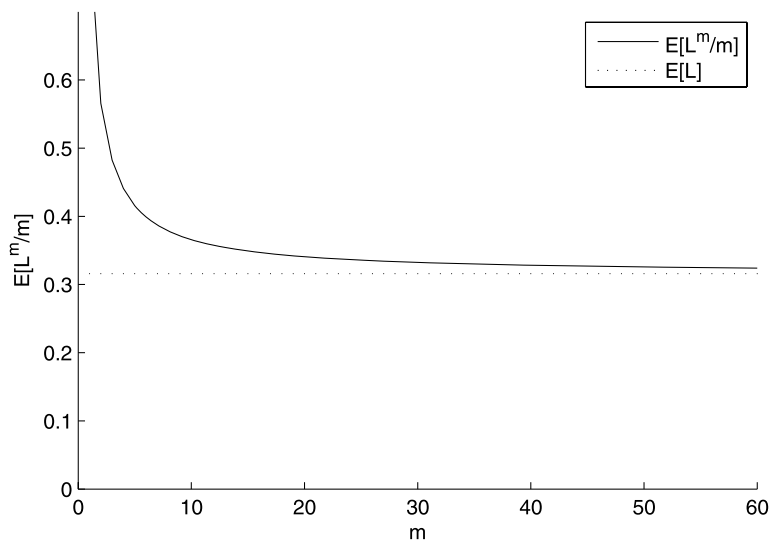




\section{Conclusion and possible extensions}

In this paper we studied the abandonment phenomenon in queueing systems with vacations, where there exist a kind of synchronization for the abandonments. More specifically, we analyzed two models with respect to the abandonment decisions of the customers (unique or multiple). We studied the stationary distributions for the number of customers in the system in continuous time for the Markovian models and we also consider some aspects of the analysis in the non-Markovian case. It would be interesting to consider extensions of this methodology for the study of other models with this type of binomial transitions.

A first direction is to carry out the analysis of the many variations of these models. For example, we can also consider the many-server case and the infinite-server case. We should then specify the way in which the servers take the vacations. The simplest case is the one where all servers take a vacation when the system becomes empty and all of them return as in the one-server case. This agrees to the many-server case with independent abandonments in the paper of Altman and Yechiali [3] . We can also consider the single-vacation case which is different from the multiple-vacation case described above in that the server takes just one vacation and then remains in the system even if there are no waiting customers present.

Open Access This article is distributed under the terms of the Creative Commons Attribution Noncommercial License which permits any noncommercial use, distribution, and reproduction in any medium, provided the original author(s) and source are credited.

\section{References}

1. Adan, I., Weiss, G.: Analysis of a simple Markovian re-entrant line with infinite supply of work under the LBFS policy. Queueing Syst. 54, 169-183 (2006)

2. Altman, E., Borovkov, A.A.: On the stability of retrial queues. Queueing Syst. 26, 343-363 (1997)

3. Altman, E., Yechiali, U.: Analysis of customers' impatience in queues with server vacations. Queueing Syst. 52, 261-279 (2006)

4. Artalejo, J.R., Economou, A., Lopez-Herrero, M.J.: Evaluating growth measures in populations subject to binomial and geometric catastrophes. Math. Biosci. Eng. 4, 573-594 (2007)

5. Baccelli, F., Boyer, P., Hebuterne, G.: Single-server queues with impatient customers. Adv. Appl. Probab. 16, 887-905 (1984)

6. Boxma, O.J., de Waal, P.R.: Multiserver queues with impatient customers. ITC 14, 743-756 (1994)

7. Daley, D.J.: General customer impatience in the queue $G I / G / 1$. J. Appl. Probab. 2, 186-205 (1965)

8. Denteneer, D., van Leeuwaarden, J.S.H., Adan, I.J.B.F.: The acquisition queue. Queueing Syst. 56(3), 229-240 (2007)

9. Economou, A.: The compound Poisson immigration process subject to binomial catastrophes. J. Appl. Probab. 41, 508-523 (2004)

10. Economou, A., Fakinos, D.: Alternative approaches for the transient analysis of Markov chains with catastrophes. J. Stat. Theory Pract. 2, 183-197 (2008)

11. Economou, A., Kapodistria, S.: Q-series in Markov chains with binomial transitions: studying a queue with synchronization. Probab. Eng. Inf. Sci. 23, 75-99 (2009)

12. Fuhrmann, S.W.: A note on the $M / G / 1$ queue with server vacations. Oper. Res. 32, 1368-1373 (1984)

13. Gasper, G., Rahman, M.: Basic Hypergeometric Series, 2nd edn. Cambridge University Press, Cambridge (2004)

14. Hanschke, X.: Explicit formulas for the characteristics of the $M / M / 2 / 2$ queue with repeated attempts. J. Appl. Probab. 24, 486-494 (1987) 
15. Ismail, M.E.H.: A queueing model and a set of orthogonal polynomials. J. Math. Anal. Appl. 108, 575-594 (1985)

16. Kemp, A.W.: Absorption sampling and absorption distribution. J. Appl. Probab. 35, 489-494 (1998)

17. Kemp, A.W.: Steady-state Markov chain models for certain $q$-confluent hypergeometric distributions. J. Stat. Plan. Inference 135, 107-120 (2005)

18. Krishnamoorthy, A., Deepak, T., Joshua, V.: An $M / G / 1$ retrial queue with nonpersistent customers and orbital search. Stoch. Anal. Appl. 23, 975-997 (2005)

19. Palm, C.: Methods of judging the annoyance caused by congestion. Tele 4, 189-208 (1953)

20. Palm, C.: Research on telephone traffic carried by full availability groups. Tele 1, 107 (1957) (English translation of results first published in 1946 in Swedish in the same journal, which was then entitled Tekniska Meddelanden fran Kungl. Telegrfstyrelsen)

21. Ross, S.M.: Introduction to Probability Models, 8th edn. Academic Press, London (2003)

22. Takacs, L.: A single-server queue with limited virtual waiting time. J. Appl. Probab. 11, 612-617 (1974)

23. Takagi, H.: Vacation and Priority Systems. Queueing Analysis-A Foundation of Performance Evaluation, vol. I. North-Holland, New York (1991)

24. Tian, N., Zhang, Z.G.: Vacation Queueing Models: Theory and Applications. Springer, New York (2006)

25. Yechiali, U.: Queues with system disasters and impatient customers when system is down. Queueing Syst. 56, 195-202 (2007) 